\title{
Generation Theorems of Semi-Groups of Linear Operators
}

By

\author{
I. Miyadera*, S. Oharu** and N. Okazawa***
}

This paper concerns the generation of semi-groups of linear operators in a Banach space $X$.

By a semi-group $\{T(t) ; t \geqq 0\}$ on $X$ we mean a one-parameter family of bounded linear operators $T(t), t \geqq 0$, such that $T(0)=I$ (the identity operator), $T(t+s)=T(t) T(s)$ for $t, s \geqq 0$ and such that for each $x \in X$, $T(t) x$ is strongly continuous in $t>0$. For a given semi-group $\{T(t) ; t$ $\geqq 0\}$ on $X$, we define the infinitesimal generator $A_{0}$ by $A_{0} x=\lim _{h \rightarrow 0+}$ $h^{-1}(T(h) x-x)$ whenever the limit exists. We wish to investigate the structure and properties of $\{T(t) ; t \geqq 0\}$ through those of $A_{0}$. While it is desirable that $A_{0}$ has nice properties, $A_{0}$ is not necessarily closed and the domain $D\left(A_{0}\right)$ is not dense in $X$ in general. In fact, a semi-group of class $(0, A)$ is of class $\left(0, C_{1}\right)$ if and only if $A_{0}$ is closed, see Phillips [11]; an interesting semi-group on $X$ with the infinitesimal generator $A_{0}$ such that $\overline{D\left(A_{0}\right)} \neq X$ is discussed in Lagnese [7]. In order to investigate the properties of $A_{0}$, we consider two kinds of modified generators. One of them will be called the infinitesimal generator in the sense of Feller and the other the complete infinitesimal generator. Our first purpose is to study the basic properties of these generators. The study of the former generator is connected to the work of Feller [3]. To consider the later generator we need to make an additional assumption on the Laplace transform of the semi-group. These generators have dense domains if and

Received August 10, 1972.

Communicated by S. Matsuura.

* Department of Mathematics, Waseda University, Tokyo, Japan.

** Same as above.

*** Department of Applied Physics, Waseda University, Tokyo, Japan. 
only if the range of the semi-group $\{T(t) ; t \geqq 0\}, X_{0}=\bigcup_{t>0} T(t)[X]$, is dense in $X$.

By the continuity set of a semi-group $\{T(t) ; t \geqq 0\}$ we mean the set $\Sigma=\left\{x \in X ; \lim _{h \rightarrow 0+} T(h) x=x\right\}$. This set plays an important role in the theory of semi-groups of linear operators and it might be natural to classify semi-groups on $X$ in terms of the continuity set. In Oharu [9] certain classes of semi-groups on $X$ are introduced. That is, for each nonnegative integer $k$, we consider a class of semi-groups $\{T(t) ; t \geqq 0\}$ on $X$ such that $D\left(A^{k}\right)$ is contained in the continuity set of $\{T(t) ; t \geqq 0\}$, where $A$ is the complete infinitesimal generator. In [9], however, $A$ has always non-empty resolvent set; such a class is called class $\left(C_{(k)}\right)$ and the fundamental classes discussed in Hille-Phillips [4] are contained in the classes $\left(C_{(k)}\right), k=0,1,2$. Our second purpose is to extend these classes to the case in which the resolvent set of the generator can be empty. In this paper those extended classes will be called classes $\left(\mathfrak{S}_{k}\right), k=0,1,2, \ldots$. As will be shown, $\left(C_{(k)}\right) \subset\left(\mathfrak{S}_{k}\right)$ and $\left(\mathfrak{S}_{k}\right) \varsubsetneqq\left(\mathfrak{S}_{k+1}\right)$ for each $k$ in the set theoretical sense. Also, those classes contain, as their important subclasses, the semi-groups of growth order $\alpha$ which have been studied by Da Prato [2] and Sobolevskii [12].

Our third and main purpose is to characterize the classes of semigroups mentioned above in terms of the corresponding infinitesimal generators. We proceed with our argument as follows: Let $A$ be a linear operator in $X$ and $Y$ be a linear subset of $X$. We then impose the following conditions:

$\left(\mathrm{a}_{1}\right) \quad Y$ is a normed space under a certain norm $\|\cdot\| \|$,

$\left(\mathrm{a}_{2}\right)$ there is a real $\omega$ such that for each $\lambda>\omega, R(\lambda-A)$ contains $Y$, $R(\lambda)=(\lambda-A)^{-1}$ exists and such that $Y$ is invariant under $R(\lambda)$,

$\left(\mathrm{a}_{3}\right)$ there exists a constant $M>0$ such that $\left\|R(\lambda)^{n} x\right\| \leqq$ $M(\lambda-\omega)^{-n}\|x\|$ for $x \in Y, \lambda>\omega$ and $n=0,1,2, \ldots$

Under these conditions there is a one-parameter family $\{T(t) ; t \geqq 0\}$ of linear operators defined on a subset $Y_{1}$ of $Y$ such that $T(t) x=\lim _{n \rightarrow \infty}$ $(I-(t / n) A)^{-n} x$ for $t \geqq 0$ and $x \in Y_{1}$. If in addition, we make some assumptions on the denseness of $Y_{1}$ in $Y,\{T(t) ; t \geqq 0\}$ can be extended 
to a semi-group of class $\left(C_{0}\right)$ on a certain Banach space $\tilde{Y}$. Moreover, we can extend this semi-group on $\tilde{Y}$ to a semi-group on $X$ by imposing a condition of Feller type.

The fundamental idea for constructing a semi-group under these conditions is based on that of Feller [3]; we first introduce into $Y$ another norm $N(\cdot)$ defined by $N(x)=\sup \left\{\left\|(\lambda-\omega)^{n} R(\lambda)^{n} x\right\| ; \lambda>\omega, n \geqq 0\right\}, x \in Y$, and then regard the resolvents $R(\lambda)$ multiplied by $(\lambda-\omega)$ as contractions in this new normed space $Y$. A quite similar method to ours is employed in a paper by Krein-Laptev-Cvetkova [6] in which the underlying operator $A$ is supposed to be closed and the Hille-Yosida theorem is applied. In this paper, the operator $A$ need not be closed and the corresponding semi-group is constructed by employing the method established by CrandallLiggett [1]. In this way, we construct semi-groups in a general way and, as the particular cases, we shall improve a Feller's theorem given in [3] and give the generation theorem for semi-groups of classes $\left(\Im_{k}\right)$.

Finally, our fourth purpose is to derive some sufficient conditions for the existence of solution operators of an abstract Cauchy problem, ACP, formulated for a closed operator in a Banach space. The results are obtained from the generation theorems mentioned above and give sufficient conditions for the Hadamard correctness of ACP which is discussed by [6]. On the other hand, Sunouchi [15] and Takahashi-Oharu [16] discussed ACP's which are well-posed in the sense of semi-group. Our results also furnish some sufficient conditions for such well-posedness.

This paper consists of seven sections. Section 1 contains some special notations used in this paper and some basic notions. Section 2 treats the infinitesimal generator in the sense of Feller. In Section 3, classes $\left(\Im_{k}\right)$ are introduced and their properties are investigated. Section 4 deals with some basic estimates for the construction of semi-groups. Section 5 concerns the construction of semi-groups and in Section 6, the generation theorems for the semi-groups discussed in Sections 2 and 3 are established. Finally, Section 7 concerns the abstract Cauchy problem. 


\section{Notations and Definitions}

In this section we list some notations and basic notions. Let $X$ and $Y$ be (complex) Banach spaces. By $B(X, Y)$ we denote the set of all bounded linear operators on $X$ into $Y$; we write $B(X)$ for $B(X, X)$. For a $U \in B(X)$ and a subset $S \subset X$, we denote the image of $S$ under $U$ by $U[S]$. Let $A$ be a linear operator from $X$ into $Y$. Then $D(A)$ and $R(A)$ stand for its domain and range, respectively. We write $\rho(A)$ for the resolvent set of $A$ and $R(\lambda ; A)$ for the resolvent of $A$ at $\lambda(\in \rho(A))$. If the null manifold of $A$ consists of only 0 , then $A^{-1}$ is defined as a linear operator from $Y$ into $X$; in this case we say that $A$ is invertible. We sometimes call a linear operator $A$ with $D(A)$ and $R(A)$ in $X$ an operator in $X$.

Let $S \subset X$ and $A$ be an operator from $X$ into $Y$. We write $A \mid S$ for the restriction of $A$ to $D(A) \cap S . \quad \bar{S}$ denotes the closure of $S$ and $\bar{A}$ stands for the closure of $A$ provided that $A$ is closable. For a closable operator $A$ such that $\bar{A}=B, D(A)$ is called a core of $B$, that is, a linear manifold $D(\subset D(B))$ is a core of $B$ if $D$ is dense in $D(B)$ with respect to the graph norm of $B$.

In this paper we consider for a fixed Banach space $X$ several kinds of Banach spaces which are contained in $X$ as its linear subsets and are endowed with stronger norms than the norm of $X$ : Let $A$ be a closed operator in $X$. Then for each positive integer $k$ we can regard $D\left(A^{k}\right)$ as a Banach space under the norm

$$
\|x\|_{k}=\sum_{i=0}^{k}\left\|A^{i} x\right\|, \quad x \in D\left(A^{k}\right)
$$

where $A^{0}=I$ (the identity operator on $X$ ); we write $\left[D\left(A^{k}\right)\right]$ for the Banach space. Accordingly, every element $U \in B(X)$ is simultaneously an element of $B\left(\left[D\left(A^{k}\right)\right], X\right)$ for each $k$. Also, we consider other spaces $\sum, \widetilde{\Sigma}$ and $Y$. Their norms are denoted by $N(\cdot), \tilde{N}(\bullet)$ and $\|\cdot\|$, respectively. These spaces are treated in a fixed Banach space and hence it is needed to specify the topologies when we discuss the problem of conver- 
gence, denseness and so on. We then specify the norm in question by putting the corresponding symbols of norm before the adjectives, nouns and symbols, such as $\|\cdot\| \|$-dense, $\|\cdot\| \cdot \|$-core, $\|\cdot\| \|-\lim _{n \rightarrow \infty} x_{n}=x$, and so on.

\section{Semi-Groups of Linear Operators}

Let $X$ be a Banach space. A one-parameter family $\{T(t) ; t \geqq 0\}$ in the Banach algebra $B(X)$ is called a semi-group (of operators) on $X$, if

$$
\begin{gathered}
T(t+s)=T(t) T(s) \quad \text { for } t, s \geqq 0, T(0)=I, \\
\text { for each } x \in X, T(t) x \text { is strongly continuous in } t>0 .
\end{gathered}
$$

In this section we discuss about the fundamental properties of semi-groups on $X$. These properties are investigated through the notion of semi-group of class $\left(C_{0}\right)$ on a certain Banach space. If $\{T(t) ; t \geqq 0\} \subset B(X)$ satisfies (2.1) and

$$
\lim _{h \rightarrow 0+} T(h) x=x \quad \text { for all } x \in X
$$

then it is called a semi-group of class $\left(C_{0}\right)$ on $X$; note that (2.2) is automatically satisfied in this case.

Let $\{T(t) ; t \geqq 0\}$ be a semi-group on $X$. Then it is proved (HillePhillips $\left[4 ;\right.$ p. 306]) that $\omega_{0}=\lim _{t \rightarrow \infty} t^{-1} \log \|T(t)\|$ exists and $-\infty \leqq \omega_{0}<$ $+\infty ; \omega_{0}$ is called the type of the semi-group. The set

$$
\Sigma=\left\{x \in X ; \lim _{h \rightarrow 0+}\|T(h) x-x\|=0\right\}
$$

is called the continuity set. We define the infinitesimal generator $A_{0}$ by

$$
A_{0} x=\lim _{h \rightarrow 0+} A_{h} x, A_{h}=h^{-1}(T(h)-I)
$$

whenever the limit exists. It is well-known that $A_{0}$ is not necessarily closed. We then introduce two kinds of modifications of infinitesimal generator. If $A_{0}$ is closable, the closure $\bar{A}_{0}$ is called the complete infinitesimal generator of the semi-group. Also, according to Feller [3], we define the operator $\Omega$ from $\Sigma$ into itself by the relations 


$$
\Omega x=A_{0} x, x \in D(\Omega)=\left\{x \in D\left(A_{0}\right) ; A_{0} x \in \Sigma\right\} ;
$$

we call this restriction of $A_{0}$ the infinitesimal generator in the sense of Feller.

First of all, we introduce a norm under which $\Sigma$ becomes a Banach space. Let $\omega\left(>\omega_{0}\right)$ be arbitrarily fixed. Since $e^{-\omega t}\|T(t)\| \rightarrow 0$ as $t \rightarrow$ $+\infty,\left\|e^{-\omega t} T(t) x\right\|$ is bounded on $[0, \infty)$ for each $x \in \Sigma$. We then define a function $N(x)$ on $\Sigma$ by

$$
N(x)=\sup _{t \geq 0}\left\|e^{-\omega t} T(t) x\right\| \quad \text { for } x \in \Sigma .
$$

It is easily seen that $\|x\| \leqq N(x)(<+\infty)$ for $x \in \Sigma$ and that $N(x)$ defines a norm on $\Sigma$. Moreover, we can prove the following (see Feller [3]).

Lemma 2.1. $\sum$ is a Banach space under the norm $N(\cdot)$.

The semi-group $\{T(t)\}$ can be regarded as a semi-group of class $\left(C_{0}\right)$ on the Banach space $\Sigma$ in the following sense: Let

$$
U(t)=T(t) \mid \Sigma \quad \text { for } t \geqq 0 .
$$

Then each $U(t)$ maps $\Sigma$ into itself and for the family of these operators $\{U(t) ; t \geqq 0\}$ we obtain

Theorem 2.2. $\{U(t) ; t \geqq 0\}$ is a semi-group of class $\left(C_{0}\right)$ on the Banach space $\Sigma$ such that

$$
N(U(t) x) \leqq e^{\omega t} N(x) \quad \text { for } t \geqq 0 \text { and } x \in \Sigma
$$

and the operator $\Omega$ defined by (2.4) coincides with the infinitesimal generator of this semi-group. Moreover, $D\left(\Omega^{\infty}\right)=\bigcap_{n \geqq 1} D\left(\Omega^{n}\right)$ is $N(\cdot)$-dense in $\Sigma$.

Proof. Clearly, the definition yields that $\{U(t)\}$ has the semi-group property (2.1). Let $x \in \sum$. Then $N(U(t) x)=\sup _{s \geq 0}\left\|e^{-\omega s} T(s) T(t) x\right\|=$ $e^{\omega t} \sup _{s \geqq 0}\left\|e^{-\omega(s+t)} T(s+t) x\right\| \leqq e^{\omega t} N(x)$ for $t \geqq 0$. Since $\lim _{s \rightarrow \infty}\left\|e^{-\omega s} T(s)\right\|$ $=0$, the function $e^{-\omega s} T(s) x$ is uniformly $\|\cdot\|$-continuous in $s \in[0, \infty)$ and 
hence $\lim _{t \rightarrow 0+} \sup _{s \geqq 0}\left\|e^{-\omega(t+s)} T(t+s) x-e^{-\omega s} T(s) x\right\|=0$. Therefore,

$$
\begin{aligned}
& N(U(t) x-x)=\sup _{s \geqq 0}\left\|e^{-\omega s} T(s)(T(t) x-x)\right\| \\
&=\sup _{s \geqq 0}\left\|\left\{e^{-\omega(t+s)} T(t+s) x-e^{-\omega s} T(s) x\right\}+\left(e^{\omega t}-1\right) e^{-\omega(t+s)} T(t+s) x\right\| \\
& \leqq \sup _{s \geqq 0}\left\|e^{-\omega(t+s)} T(t+s) x-e^{-\omega s} T(s) x\right\|+\left(e^{\omega t}-1\right) N(x) \rightarrow 0
\end{aligned}
$$

as $t \rightarrow 0+$, which means that $\{U(t)\}$ forms a semi-group of class $\left(C_{0}\right)$ on $\sum$. In view of (2.5) and (2.6), $\left\{x \in X ; \exists N(\cdot)-\lim _{h \rightarrow 0+} h^{-1}(U(h) x-\right.$ $x)\} \subset D(\Omega)$. To show the converse relation of the implication, let $x \in D(\Omega)$. Since $\Omega x \in \sum$ and since $T(t) \Omega x$ is the continuous derivative of $T(t) x$, it follows that $t^{-1}\{T(t+s) x-T(s) x\}=t^{-1} \int_{s}^{s+t} T(\tau) \Omega x d \tau$ for $s \geqq 0$ and $t>0$. In view of this, we have

$$
\begin{aligned}
& N\left(t^{-1}[U(t) x-x]-\Omega x\right)=\sup _{s \geqq 0}\left\|e^{-\omega s} T(s)\left\{t^{-1}[T(t) x-x]-\Omega x\right\}\right\| \\
& \quad=\sup _{s \geqq 0}\left\|e^{-\omega s} t^{-1} \int_{s}^{s+t} T(\tau) \Omega x d \tau-e^{-\omega s} t^{-1} \int_{s}^{s+t} T(s) \Omega x d \tau\right\| \\
& \leqq t^{-1} \sup _{s \geqq 0} \int_{s}^{s+t}\left\|e^{-\omega \tau} T(\tau) \Omega x-e^{-\omega s} T(s) \Omega x\right\| d \tau \\
& \quad+t^{-1} \sup _{s \geqq 0} \int_{s}^{s+t}\left(e^{\omega(\tau-s)}-1\right)\left\|e^{-\omega \tau} T(\tau) \Omega x\right\| d \tau .
\end{aligned}
$$

Since $e^{-\omega s} T(s) \Omega x$ is uniformly $\|\cdot\|$-continuous on $[0, \infty)$, given an $\varepsilon>0$ there is a $\delta=\delta(\varepsilon)>0$ such that $\left\|e^{-\omega \tau} T(\tau) \Omega x-e^{-\omega s} T(s) \Omega x\right\|<\varepsilon / 2$ and $N(\Omega x)\left|e^{\omega(\tau-s)}-1\right|<\varepsilon / 2$ provided $|\tau-s| \leqq \delta$. Consequently, $N\left(t^{-1}\{U(t) x\right.$ $-x\}-\Omega x) \leqq \varepsilon$ for $t \in(0, \delta)$. This implies that $\Omega$ becomes the infinitesimal generator of $\{U(t)\}$. Finally, the last assertion is well-known for the semi-group of class $\left(C_{0}\right)$, see Hille-Phillips [4; Theorem 10.3.4].

Q.E.D.

In the remainder of this section we are concerned with the Laplace transform of a semi-group $\{T(t) ; t \geqq 0\}$ on $X$. For each $\lambda\left(>\omega>\omega_{0}\right)$, we can define an operator $J(\lambda)$ on $\Sigma$ by

$$
J(\lambda) x=\int_{0}^{\infty} e^{-\lambda t} T(t) x d t\left(=\int_{0}^{\infty} e^{-\lambda t} U(t) x d t\right) \quad \text { for } x \in \Sigma .
$$


In view of Theorem 2.2, we see that the integral converges with respect to $N(\cdot)$-norm and a fortiori the original $\|\cdot\|$-norm. We list, as a Corollary to Theorem 2.2, some of the fundamental properties of $J(\lambda)$ which are well-known in the general theory of semi-groups of class $\left(C_{0}\right)$.

Corollary 2.3. (i) $\Omega$ is a closed operator in $\sum$ and for each $\lambda>\omega$, $J(\lambda)$ is the resolvent of $\Omega$, i.e., $J(\lambda)=(\lambda-\Omega)^{-1} \in B(\Sigma)$.

(ii) $N(J(\lambda) x) \leqq(\lambda-\omega)^{-1} N(x) \quad$ for $\lambda>\omega$ and $x \in \Sigma$.

(iii) $N(\cdot)-\lim _{\lambda \rightarrow+\infty} \lambda J(\lambda) x=x \quad$ for $x \in \Sigma$.

(iv) $J(\lambda)^{n} x=(n-1) !^{-1} \int_{0}^{\infty} e^{-\lambda s} s^{n-1} U(s) x d s$ for $x \in \sum, \lambda>\omega$ and $n=1,2,3, \ldots$

(v) $D\left(\Omega^{\infty}\right)$ is an $N(\cdot)$-core of $\Omega$.

Proof. The proofs of (i)-(iv) are stated in Hille-Phillips [4; Theorem 12. 3. 1]. (v) follows from the last assertion of Theorem 2.2 and (ii). See also Oharu $[9 ;$ Lemma 3.6$]$.

Q.E.D.

In view of this corollary and by the same argument as in Feller [3; Section 3], we obtain the following

Theorem 2.4. (i) $N(x)=\sup _{n \geqq 0, \lambda>\omega}\left\|(\lambda-\omega)^{n} J(\lambda)^{n} x\right\|$ for $x \in \Sigma$.

(ii) For any $\varepsilon>0$ and $x \in \sum$, there exist $K_{\varepsilon}>0$ and $\lambda_{0}=\lambda_{0}(\varepsilon, x)$ such that

$$
\left\|(\lambda-\omega)^{n} J(\lambda)^{n} x\right\| \leqq K_{\varepsilon}\|x\| \quad \text { for } \lambda>\lambda_{0} \text { and } n \geqq(\lambda-\omega) \varepsilon .
$$

Remark 2.5. Condition (ii) is the so-called condition of Feller type. This condition implies the following (ii') which is proposed in Oharu $[9$; Section 5]:

(ii') For any $\varepsilon>0$ and $x \in \sum$, there exist $M_{\varepsilon}>0$ and $\lambda_{0}^{\prime}=\lambda_{0}^{\prime}(\varepsilon, x)$ such that 


$$
\left\|\lambda^{n} J(\lambda)^{n} x\right\| \leqq M_{\varepsilon}\|x\| \text { for } \lambda>\lambda_{0}^{\prime} \text { and } n \text { with } n / \lambda \in[\varepsilon, 1 / \varepsilon] \text {. }
$$

(ii') is obtained from Theorem 2.4 (ii) as follows: Let $x \in \Sigma$ and $\varepsilon>0$. Then $\left\|\lambda^{n} J(\lambda)^{n} x\right\|=|1-\omega / \lambda|^{-n}\left\|(\lambda-\omega)^{n} J(\lambda)^{n} x\right\|$ for $n \geqq 1$ and $\lambda>\omega$. We then set $\lambda_{0}^{\prime}=\max \left\{\lambda_{0}(\varepsilon / 2, x), 2|\omega|\right\}$ and $M_{\varepsilon}=\exp (2|\omega| / \varepsilon) K_{\varepsilon / 2}$, where $\lambda_{0}(\varepsilon / 2, x)$ and $K_{\varepsilon / 2}$ are the constants associated with $x$ and $\varepsilon / 2$ through Theorem 2.4 (ii). If $\lambda>\lambda_{0}^{\prime}$ and $n / \lambda \in[\varepsilon, 1 / \varepsilon]$, then by Theorem 2.4 (ii), $|1-\omega / \lambda|^{-n} \leqq \exp (2|\omega| / \varepsilon)$ and $\left\|(\lambda-\omega)^{n} J(\lambda)^{n} x\right\| \leqq K_{\varepsilon / 2}\|x\|$. Note that $\lambda>\lambda_{0}(\varepsilon / 2, x)(>\omega)$ and $n \geqq \lambda \varepsilon \geqq(\lambda-\omega) \varepsilon / 2$. Consequently, we have $\left\|\lambda^{n} J(\lambda)^{n} x\right\| \leqq \exp (2|\omega| / \varepsilon) K_{\varepsilon / 2}\|x\|=M_{\varepsilon}\|x\|$ for $\lambda>\lambda_{0}^{\prime}$ and $n / \lambda \in[\varepsilon, 1 / \varepsilon]$.

Finally, the following theorem due to Feller [3] is obtained by combining the results obtained so far.

Theorem 2.6. Let $\{T(t) ; t \geqq 0\}$ be a semi-group on $X$ such that $\bigcup_{t>0} T(t)[X]$ is dense in $X$. Then we have:

(a) the continuity set $\Sigma$ is dense in $X$.

(b) $\Sigma$ is a Banach space under the norm $N(\cdot)$.

(c) $\Omega$ defined by (2.4) is $N(\cdot)$-densely defined in $\Sigma$.

(d) For each $\lambda>\omega$ and $x \in \sum$ the equation $(\lambda-\Omega) y=x$ has a unique solution $y=J(\lambda) x=\int_{0}^{\infty} e^{-\lambda t} T(t) x d t$.

(e) $N(x)=\sup _{n \geqq 0}, \lambda>\omega\left\|(\lambda-\omega)^{n} J(\lambda)^{n} x\right\| \quad$ for $x \in \Sigma$.

(f) For any $\varepsilon>0$ and $x \in \sum$, there exist $K_{\varepsilon}>0$ and $\lambda_{0}=\lambda_{0}(\varepsilon, x)$ such that $\left\|(\lambda-\omega)^{n} J(\lambda)^{n} x\right\| \leqq K_{\varepsilon}\|x\|$ for $\lambda>\lambda_{0}$ and $n \geqq(\lambda-\omega) \varepsilon$.

\section{Semi-Groups of Class $\left(\mathfrak{S}_{k}\right)$}

In this section we first investigate the closability of the infinitesimal generator of a semi-group on $X$ and then introduce some classes of semigroups on $X$. Also, some of their basic properties are given.

Let $X$ be a Banach space and $\{T(t) ; t \geqq 0\}$ be a semi-group on $X$ with the type $\omega_{0}$ and with the infinitesimal generator $A_{0}$. We set

$$
X_{0}=\bigcup_{t>0} T(t)[X]
$$

and for each $\lambda>\omega_{0}$, we define an operator $R_{0}(\lambda)$ on $X_{0}$ by 


$$
R_{0}(\lambda) x=\int_{0}^{\infty} e^{-\lambda t} T(t) x d t \quad \text { for } x \in X_{0}
$$

Clearly, $X_{0}$ is contained in the continuity set $\Sigma$ of $\{T(t)\}$ and $R_{0}(\lambda) C$ $J(\lambda)$ for $\lambda>\omega\left(>\omega_{0}\right)$, where $J(\lambda)$ is the operator defined on $\Sigma$ by (2.8). To obtain the closability of $A_{0}$ we impose the following condition:

$\left(\alpha_{1}\right)$ there exists an $\omega>\omega_{0}$ such that for each $\lambda>\omega, R_{0}(\lambda)$ is closable and its closure $R(\lambda)$ is invertible.

Lemma 3.1. Assume condition $\left(\alpha_{1}\right)$. Then we have:

(i) $R(\lambda) x=\int_{0}^{\infty} e^{-\lambda t} T(t) x d t \quad$ for $x \in \sum$ and $\lambda>\omega$

(ii) $A_{0}$ is closable and its closure $A$ is equal to $\lambda-R(\lambda)^{-1}$ for every $\lambda>\omega$, i.e., $R(\lambda)=(\lambda-A)^{-1}$ for $\lambda>\omega$.

Conversery, if $A_{0}$ is closable and $\lambda-A$ is invertible for $\lambda>\omega$, where $A=$ $\bar{A}_{0}$, then condition $\left(\alpha_{1}\right)$ holds.

Proof. Suppose condition $\left(\alpha_{1}\right)$ holds. Let $\lambda>\omega$ and $x \in \sum$. Then $R(\lambda) T(s) x=\int_{0}^{\infty} e^{-\lambda t} T(t+s) x d t=e^{\lambda s} \int_{s}^{\infty} e^{-\lambda t} T(t) x d t \rightarrow \int_{0}^{\infty} e^{-\lambda t} T(t) x d t$ as $s \rightarrow 0+$. Since $\lim _{s \rightarrow 0+} T(s) x=x$, the closedness of $R(\lambda)$ implies that $x \in D(R(\lambda)$ ) and that the relation (i) holds. In view of this, we have

$$
A_{h} R(\lambda) x=R(\lambda) A_{h} x=h^{-1}\left(e^{\lambda h}-1\right) R(\lambda) x-e^{\lambda h} h^{-1} \int_{0}^{h} e^{-\lambda t} T(t) x d t
$$

hence, passing to the limit as $h \rightarrow 0+$,

$$
A_{0} R(\lambda) x=\lim _{h \rightarrow 0+} A_{h} R(\lambda) x=\lim _{h \rightarrow 0+} R(\lambda) A_{h} x=\lambda R(\lambda) x-x .
$$

From this and the closedness of $R(\lambda)$, it follows that $A_{0} x \in D(R(\lambda))$ and

$$
R(\lambda) A_{0} x=\lambda R(\lambda) x-x
$$

provided that $x \in D\left(A_{0}\right)$. To show the closability of $A_{0}$, let $x_{n} \in D\left(A_{0}\right)$, $x_{n} \rightarrow 0$ and $A_{0} x_{n} \rightarrow y$ as $n \rightarrow \infty$. Then, $R(\lambda)\left(\lambda-A_{0}\right) x_{n}=x_{n} \rightarrow 0$ by (3.3) and hence $R(\lambda)(-y)=0$. Since $R(\lambda)$ is invertible, we obtain $y=0$. This 
means that $A_{0}$ is closable. Now, writing $A$ for the closure $\bar{A}_{0}$ and then using the relation (3.3), we see that $(\lambda-A) x \in D(R(\lambda))$ and $R(\lambda)(\lambda-A) x$ $=x$ for $x \in D(A)$. Next, let $x \in D(R(\lambda))$. Then there exist $x_{n} \in X_{0}$ such that $x_{n} \rightarrow x$ and $R_{0}(\lambda) x_{n} \rightarrow R(\lambda) x$ as $n \rightarrow \infty$. Since $(\lambda-A) R(\lambda) x_{n}=(\lambda-$ $\left.A_{0}\right) R(\lambda) x_{n}=x_{n}$ by (3.2), the closedness of $(\lambda-A)$ implies that $R(\lambda) x \in$ $D(A)$ and $(\lambda-A) R(\lambda) x=x$. Consequently, $R(\lambda)=(\lambda-A)^{-1}$. Since $\lambda$ was arbitrary as far as $\lambda>\omega$, we have the assertion (ii). Finally, we show the converse. Suppose that $A_{0}$ is closable and that $\lambda-A\left(=\lambda-\bar{A}_{0}\right)$ is invertible for $\lambda>\omega$. In the same way as in (3.2), we obtain

$$
(\lambda-A) R_{0}(\lambda) x=\left(\lambda-A_{0}\right) R_{0}(\lambda) x=x \quad \text { for } x \in X_{0} \text { and } \lambda>\omega .
$$

In view of this, we see using the closedness of $A$ and then applying the invertibility of $\lambda-A$ that $R_{0}(\lambda)$ is closable and that $(\lambda-A) R(\lambda) x=x$ for each $x \in D(R(\lambda))$. This shows that $R(\lambda)$ is invertible provided $\lambda>\omega$.

Q.E.D.

We now exibit a relationship between the complete infinitesimal generator $\bar{A}_{0}$ and the infinitesimal generator in the sense of Feller $\Omega$.

Lemma 3.2. Assume condition $\left(\alpha_{1}\right)$. Then $\Omega$ is closable as an operator in $X$ and its closure coincides with the complete infinitesimal generator, i.e., $A=\bar{A}_{0}=\bar{\Omega}$.

Proof. We first note that $A=\bar{A}_{0}$ exists by Lemma 3.1. Since $\Omega \subset$ $A_{0} \subset A, \Omega$ is closable and $\bar{\Omega} \subset A$. We want to show that $D(A)$ (= the range of $R(\lambda)) \subset D(\bar{\Omega})$. Let $\lambda>\omega$. Then by (3.2) $A_{h} R(\lambda) x \rightarrow \lambda R(\lambda) x-$ $x \in \sum$ as $h \rightarrow 0$ for each $x \in \sum$, which means that $R(\lambda) x \in D(\Omega)$ and $\Omega R(\lambda) x=\lambda R(\lambda) x-x$, i.e.,

$$
(\lambda-\bar{\Omega}) R(\lambda) x=(\lambda-\Omega) R(\lambda) x=x \quad \text { for } x \in \Sigma .
$$

Now, we take an arbitrary element $x \in D(R(\lambda))$. Then there exist $x_{n} \in$ $X_{0}$ such that $\left(x_{n}, R_{0}(\lambda) x_{n}\right) \rightarrow(x, R(\lambda) x)$ in $X \times X$ as $n \rightarrow \infty$. But, $(\lambda-\Omega)$ $R(\lambda) x_{n}=x_{n}$ by (3.4); hence it follows that $R(\lambda) x \in D(\bar{\Omega})$. This states that $D(A) \subset D(\bar{\Omega})$.

Q.E.D. 
Now, we introduce the following classes of semi-groups on $X$ :

Definition 3.3. Let $k$ be a nonnegative integer. A semi-group $\{T(t) ; t \geqq 0\}$ on $X$ is said to be of class $\left(\Im_{k}\right)$, if it satisfies the following conditions:

$\left(\alpha_{1}\right)$ there exists an $\omega>\omega_{0}$ such that for each $\lambda>\omega, R_{0}(\lambda)$ is closable and its closure $R(\lambda)$ is invertible;

$\left(\alpha_{2}\right) \quad X_{0}=\bigcup_{t>0} T(t)[X]$ is dense in $X$;

$\left(\alpha_{3}\right) \quad D\left(A^{k}\right) \subset \sum$, where $A$ is the complete infinitesimal generator of $\{T(t) ; t \geqq 0\}$.

Remarks. (1) In Oharu [9], some restricted classes, called classes $\left(C_{(k)}\right), k=0,1,2, \ldots$, are discussed (cf. Remark 6.5):

A semi-group $\{T(t) ; t \geqq 0\}$ on $X$ is said to be of class $\left(C_{(k)}\right)$ if it is a semi-group of class $\left(\Im_{k}\right)$ and if the operators $R(\lambda), \lambda>\omega$, are defined on $X$.

As is seen from the definition, $\left(C_{(k)}\right) \subset\left(C_{(k+1)}\right),\left(\mathfrak{S}_{k}\right) \subset\left(\mathfrak{S}_{k+1}\right)$ and $\left(C_{(k)}\right) \subset\left(\Im_{k}\right)$ for each $k$ in the set theoretical sense. We can find some examples which show that $\left(C_{(k)}\right) \varsubsetneqq\left(C_{(k+1)}\right)$ for each $k$, see $[9$; Example 4.10]. From this we see that $\left(\mathfrak{S}_{k}\right) \varsubsetneqq\left(\Im_{k+1}\right)$ for each $k$. Also, we note that classes $\left(C_{(k)}\right)$ contain the fundamental classes $(0, A)$ and $(A)$ which are discussed in Hille-Phillips [4; Section 10.6]; $\left(C_{0}\right)=\left(C_{(0)}\right)=\left(\Im_{0}\right),(0, A)$ $C\left(C_{(1)}\right)$ and $(A) \subset\left(C_{(2)}\right)$. In fact, the generation theorem for semi-groups of class $(A)$ is obtained as a corollary of that for semi-groups of class $\left(C_{(2)}\right)([9 ;$ Theorem 6.14$])$ and it is shown by Miyadera [8] that the generation theorem for semi-groups of class $(0, A)$ is obtained in view of that for semi-groups of class $\left(C_{(1)}\right)$.

(2) Da Prato [2] and then Sobolevskii [12] studied the behavior of a semi-group on $X$ at $t=0$ through the notion of growth order. Let $\alpha>$ 0 . Then a semi-group $\{T(t) ; t \geqq 0\}$ on $X$ is said to be of growth order $\alpha$ if it has the following properties:

(i) if $T(t) x=0$ for all $t>0$ then $x=0$;

(ii) $\left\|t^{\alpha} T(t)\right\|=O(1)$ as $t \rightarrow 0+$; 
(iii) $X_{0}=\bigcup_{t>0} T(t)[X]$ is dense in $X$.

Condition (i) is used to guarantee the closability of the infinitesimal generator $A_{0}$ of the semi-group and also the invertibility of $\lambda-\bar{A}_{0}, \lambda>\omega_{0}$. Under condition (ii), it is proved that $D\left(A^{[\alpha]+1}\right)$ is contained in the continuity set $\Sigma$. Consequently, a semi-group of growth order $\alpha$ is proved to be of class $\left(\mathfrak{S}_{k}\right), k=[\alpha]+1$. The detailed arguments concerning these propositions will be done in the forthcoming paper by Okazawa [10].

Now, let $\{T(t) ; t \geqq 0\}$ be a semi-group of class $\left(\Im_{k}\right)$ and let $A$ be its complete infinitesimal generator. Then $D\left(A^{k}\right)$ becomes a Banach space under the norm $\|\cdot\|_{k}$; we denote this Banach space by $\left[D\left(A^{k}\right)\right]$. Moreover, we denote by $N(x)$ the norm on the continuity set $\Sigma$ defined by (2.5) and we mean by $\omega$ the constant stated in condition $\left(\alpha_{1}\right)$.

Lemma 3.4. If $\{T(t) ; t \geqq 0\}$ is a semi-group of class $\left(\mathfrak{S}_{k}\right)$, then there exists a constant $M>0$ such that

$$
\left\|R(\lambda)^{n} x\right\| \leqq M(\lambda-\omega)^{-n}\|x\|_{k} \quad \text { for } x \in D\left(A^{k}\right), \lambda>\omega \text { and } n \geqq 0 .
$$

Proof. By the definition of $J(\lambda)$ and Lemma 3.1 (i), we see that $R(\lambda) x=J(\lambda) x$ for $\lambda>\omega$ and $x \in \sum$. Thus, by Theorem 2.4 (i),

$$
\sup _{n \geqq 0, \lambda>\omega}\left\|(\lambda-\omega)^{n} R(\lambda)^{n} x\right\| \leqq N(x) \quad \text { for } x \in D\left(A^{k}\right)\left(C \sum\right) .
$$

Now, we observe that $\left\|e^{-\omega t} T(t) x\right\| \leqq e^{-\omega t}\|T(t)\|\|x\|_{k}$ for $t>0$ and $x \in D\left(A^{k}\right)$; hence $e^{-\omega t} T(t), t>0$, are bounded operators from the Banach space $\left[D\left(A^{k}\right)\right]$ into $X$. Since $\sup _{t>0}\left\|e^{-\omega t} T(t) x\right\|(=N(x))<\infty$ for each $x \in D\left(A^{k}\right)$, the uniform boundedness theorem states that there exists an $M>0$ such that

$$
N(x)=\sup _{t>0}\left\|e^{-\omega t} T(t) x\right\| \leqq M\|x\|_{k} \quad \text { for } x \in D\left(A^{k}\right)
$$

Q.E.D.

We now obtain the following main theorem in this section.

Theorem 3.5. Let $\{T(t) ; t \geqq 0\}$ be a semi-group of class $\left(\mathfrak{S}_{k}\right)$. 
Then, it has the complete infinitesimal generator $A$ with the following properties:

(i) $D(A)$ is dense in $X$ and $D(A) \supset D\left(A_{0}\right) \supset D\left(A^{k+1}\right)$,

(ii) there is a real constant $\omega$ such that $R(\lambda-A) \supset D\left(A^{k}\right)$ and $R(\lambda)$ $=(\lambda-A)^{-1}$ exists for $\lambda>\omega$,

(iii) there is a constant $M>0$ such that

$$
\left\|R(\lambda)^{n} x\right\| \leqq M(\lambda-\omega)^{-n}\|x\|_{k}
$$

for $x \in D\left(A^{k}\right), \lambda>\omega$ and $n \geqq 0$,

(iv) for any $\varepsilon>0$ and $x \in D\left(A^{k}\right)$ there exist $M_{\varepsilon}>0$ and $\lambda_{0}=\lambda_{0}(\varepsilon, x)$ such that

$$
\left\|[\lambda R(\lambda)]^{n} x\right\| \leqq M_{\varepsilon}\|x\|
$$

for $\lambda>\lambda_{0}$ and $n$ with $n / \lambda \in[\varepsilon, 1 / \varepsilon]$,

(v) $D\left(A^{k+1}\right)$ is a core of $A$,

(vi) $D\left(A^{k+1}\right)$ is $N(\cdot)$-dense in $D\left(A^{k}\right)$, where $N(x)=\sup _{s>0}\left\|e^{-\omega s} T(s) x\right\|$ $=\sup _{n \geqq 0}, \lambda>\omega \mid(\lambda-\omega)^{n} R(\lambda)^{n} x \|, x \in D\left(A^{k}\right)$.

Proof. Condition $\left(\alpha_{2}\right)$ and the last assertion of Theorem 2.2 yield that $D(A)$ is dense in $X$. Also, by (3.2), we have that $R(\lambda)\left[\sum\right] \subset D\left(A_{0}\right)$. Since $\sum \supset D\left(A^{k}\right)$ and since $R(\lambda)\left[D\left(A^{k}\right)\right]=D\left(A^{k+1}\right)$, we obtain $D\left(A^{k+1}\right) C$ $D\left(A_{0}\right)$. Hence, we have the assertion (i). Lemma 3.1 states that $R(\lambda-$ $A)=D(R(\lambda)) \supset \sum$ for $\lambda>\omega$. The existence of $(\lambda-A)^{-1}$ follows from Lemma 3.1 (ii). Thus, (ii) is obtained. Lemma 3.4 gives the assertion (iii). In view of the fact that $R(\lambda)^{n} x=J(\lambda)^{n} x$ for $x \in \Sigma, \lambda>\omega$ and $n \geqq$ 1, (iv) follows from Theorem 2.4, Remark 2.5 and condition $\left(\alpha_{3}\right)$. (v) is proved by applying Corollary $2.3(\mathrm{v})$ and Lemma 3.2 , note that $D\left(\Omega^{\infty}\right) C$ $D\left(A^{k+1}\right)$. Finally, (vi) is obtained by employing the last assertion of Theorem 2.2, Theorem 2.4(i) and condition $\left(\alpha_{3}\right)$.

Q.E.D.

\section{Preliminaries for the Generation of Semi-Groups}

Let $A$ be a linear operator in $X$ and let $Y$ be a linear subset of $X$. 
We impose them the following conditions:

$\left(\mathrm{a}_{1}\right) \quad Y$ is a normed space under a certain norm $\|\cdot\| \|$ which is stronger than the original norm $\|\cdot\|$ of $X$;

$\left(\mathrm{a}_{2}\right)$ there exists a real $\omega$ such that for each $\lambda>\omega, R(\lambda-A)$ contains $Y, R(\lambda)=(\lambda-A)^{-1}$ exists, and such that $Y$ is invariant under $R(\lambda)$;

$\left(\mathrm{a}_{3}\right)$ there exists a constant $M>0$ such that

$$
\left\|R(\lambda)^{n} x\right\| \leqq M(\lambda-\omega)^{-n}\|x\|
$$

for $x \in Y, \lambda>\omega$ and $n=0,1,2, \ldots$

These conditions are generalizations of the conditions given in Theorems 2.6 and 3.5 , in the following sense:

$\left.1^{\circ}\right)$ If $\sum$ is the continuity set of a semi-group $\{T(t) ; t \geqq 0\}$ on $X$ and if $\Omega$ and $N(x)$ are defined by (2.4) and (2.5), then $\Omega$ and $\Sigma$ satisfy conditions $\left(\mathrm{a}_{1}\right)-\left(\mathrm{a}_{3}\right)$ with $\sum=Y, \Omega=A$ and $N(x)=\|x\|$ (see Theorem 2.6).

$2^{\circ}$ ) If $A$ is the complete infinitesimal generator of a semi-group of class $\left(\mathfrak{S}_{k}\right)$, then $A$ and $\left[D\left(A^{k}\right)\right]$ satisfy $\left(\mathrm{a}_{1}\right)-\left(\mathrm{a}_{3}\right)$ with $\left[D\left(A^{k}\right)\right]=Y$ and $\|x\|_{k}=\|x\|$ (see Theorem 3.5).

In this section we let $A$ and $Y$ be a pair of linear operator and linear subset of $X$ satisfying $\left(\mathrm{a}_{1}\right)-\left(\mathrm{a}_{3}\right)$ and we are concerned with the convergence of $\left(I-\frac{t}{n} A\right)^{-n}$ on $Y$ as $n \rightarrow \infty$. The results obtained here will be applied to the generation problem of semi-groups on $X$. Also, we can apply them to construct solution operators of abstract Cauchy problems. The results in this and the next sections are closely connected with those by Krein-Laptev-Cvetkova [6]; the norm $N(\cdot)$ defined by (5.2) is the same thing as the norm $\|\cdot\|_{G}$ treated in their paper and our Lemma 4.3 is similar to their Lemma 2. However we note that the operator $A$ treated in their paper is always closed, while our result is obtained without assuming the closedness of operator $A$. For the relation between our result and theirs, we shall mention it in the final section.

4.1. This subsection contains a basic estimate for the iteration of the resolvents $\left(I-\frac{t}{n} A\right)^{-n}, n=0,1,2, \ldots$ Noting that

$$
R(\lambda) x-R(\mu) x=-(\lambda-\mu) R(\lambda) R(\mu) x \quad \text { for } \lambda, \mu>\omega \text { and } x \in Y
$$


we start from the following

Lemma 4.1. Let $\lambda>\omega$. If $|h|<\lambda-\omega$, then for every positive integer $n$,

$$
R(\lambda+h)^{n} x=\sum_{l=n-1}^{\infty} C_{n-1}(-h)^{l-n+1} R(\lambda)^{l+1} x, \quad x \in Y .
$$

Proof. Condition $\left(\alpha_{3}\right)$ guarantees that the series of the right side in (4.2) is absolutely convergent with respect to the norm $\|\cdot\|$ for $n \geqq 1$ and $x \in Y$. (4.2) is proved by induction: Let $x \in Y$ and put $y=R(\lambda+h) x$. Then $y \in Y \cap D(A)$ and $(\lambda-A) y=x-h y \in Y$. Now,

$$
\begin{aligned}
\sum_{l=0}^{\infty}(-h)^{l} R(\lambda)^{l+1} x & =\sum_{l=0}^{\infty}(-h)^{l} R(\lambda)^{l+1}(\lambda-A) y-\sum_{l=0}^{\infty}(-h)^{l+1} R(\lambda)^{l+1} y \\
& =y=R(\lambda+h) x .
\end{aligned}
$$

This means that (4.2) holds for $n=1$. Suppose that (4.2) is true for $n$. Then

$$
\begin{aligned}
& \sum_{l=n}^{\infty} C_{n}(-h)^{l-n} R(\lambda)^{l+1} x \\
& \quad=\sum_{l=n l}^{\infty} C_{n}(-h)^{l-n} R(\lambda)^{l} y-\sum_{l=n}^{\infty} C_{n}(-h)^{l-n+1} R(\lambda)^{l+1} y \\
& \quad=R(\lambda)^{n} y+\sum_{l=n}^{\infty}\left[l+1 C_{n}-{ }_{l} C_{n}\right](-h)^{l-n+1} R(\lambda)^{l+1} y \\
& \quad=\sum_{l=n-1}^{\infty} C_{n-1}(-h)^{l-n+1} R(\lambda)^{l+1} y=R(\lambda+h)^{n} y=R(\lambda+h)^{n+1} x,
\end{aligned}
$$

which shows that (4.2) holds for $n+1$, too.

Q.E.D.

Corollary 4.2. If $\lambda>\mu>\omega$, then

$$
R(\mu)^{n} x=\sum_{l=n-1}^{\infty} C_{n-1}(\lambda-\mu)^{l-n+1} R(\lambda)^{l+1} x \quad \text { for } x \in Y \text { and } n \geqq 1 \text {. }
$$

Proof. Since $-(\lambda-\omega)<\mu-\lambda<0$, we have the assertion by setting $h=\mu-\lambda$ in (4.2).

Q.E.D.

Now, let us define a function $N(x)$ on $Y$ by

$$
N(x)=\sup _{n \geqq 0, \mu>\omega}\left\|(\mu-\omega)^{n} R(\mu)^{n} x\right\|, \quad x \in Y .
$$


Clearly, $N(\cdot)$ defines a norm on $Y$ such that $\|x\| \leqq N(x) \leqq M\|x\|$ for $x \in Y$. Moreover, we can obtain the following result which is crucial in our argument:

Lemma 4.3. For every $\lambda>\omega$,

$$
N((\lambda-\omega) R(\lambda) x) \leqq N(x), \quad x \in Y \text {. }
$$

Proof. We prove that

$$
\left\|(\mu-\omega)^{n} R(\mu)^{n}(\lambda-\omega)^{m} R(\lambda)^{m} x\right\| \leqq N(x)
$$

for $x \in Y, \lambda, \mu>\omega$ and $m, n \geqq 0 ;(4.4)$ is obtained by taking $m=1$ in (4.5). First, (4.5) is clearly true in case $n=0$. Next, by $(4.1), R(\lambda) R(\mu) x=$ $R(\mu) R(\lambda) x$ for $x \in Y$ and $\lambda, \mu>\omega$. Hence, it suffices to show that (4.5) holds for $x \in Y, \lambda>\mu>\omega, m \geqq 0$ and $n \geqq 1$. Let $x \in Y$ and $\lambda>\mu>\omega$. Since $R(\lambda)^{m} x \in Y$ for $m \geqq 0$ by $\left(\mathrm{a}_{2}\right)$, Corollary 4.2 yields that

$$
R(\mu)^{n} R(\lambda)^{m} x=\sum_{l=n-1}^{\infty} C_{n-1}(\lambda-\mu)^{l-n+1} R(\lambda)^{l+m+1} x
$$

for $m \geqq 0$ and $n \geqq 1$; hence

$$
\begin{gathered}
\left\|(\mu-\omega)^{n} R(\mu)^{n}(\lambda-\omega)^{m} R(\lambda)^{m} x\right\| \\
\leqq\left(\frac{\mu-\omega}{\lambda-\omega}\right)^{n} \sum_{l=n-1}^{\infty} C_{n-1}\left(1-\frac{\mu-\omega}{\lambda-\omega}\right)^{l-n+1}\left\|(\lambda-\omega)^{l+m+1} R(\lambda)^{l+m+1} x\right\| \\
\leqq\left(\frac{\mu-\omega}{\lambda-\omega}\right)^{n} \sum_{l=n-1}^{\infty} C_{n-1}\left(1-\frac{\mu-\omega}{\lambda-\omega}\right)^{l-n+1} N(x)=N(x)
\end{gathered}
$$

for $m \geqq 0$ and $n \geqq 1$.

Q.E.D.

4.2. We shall deal with the convergence of $\left(I-\frac{t}{n} A\right)^{-n}$ as $n \rightarrow \infty$. To do this, we further introduce two linear subsets of $Y$;

$$
\begin{aligned}
& Y_{1}=\{x \in Y ; A x \in Y\} \\
& Y_{2}=\left\{x \in Y_{1} ; A x \in Y_{1}\right\}
\end{aligned}
$$

From the relation (4.1), we see that 


$$
\lambda R(\lambda) x-x=R(\lambda) A x(\in Y), \quad \text { for } x \in Y_{1} \text { and } \lambda>\omega .
$$

Also, for each $t \geqq 0$ and positive integer $n$ with $n>|\omega| t$, we can define an operator $T(t ; n)$ on $Y$ by

$$
T(t ; n) x= \begin{cases}\left(I-\frac{t}{n} A\right)^{-n} x=\left\{\frac{n}{t} R\left(\frac{n}{t}\right)\right\}^{n} x & (t>0) \\ x & (t=0)\end{cases}
$$

Now, in view of Lemma 4.3, we can employ the method given by Crandall-Liggett [1] and obtain

Lemma 4.4. Let $t \geqq 0$ and $m, n$ be positive integers with $n \geqq m>$ $2|\omega| t$. Then for every $x \in Y_{1}$

$$
N(T(t ; n) x-T(t ; m) x) \leqq 2 e^{4|\omega| t}(1 / m-1 / n)^{1 / 2} N(A x) .
$$

Lemma 4.5. For each $t \geqq 0$ and integer $n$ with $n>|\omega| t$, we have:

$$
\begin{array}{ll}
N(T(t ; n) x) \leqq(1-\omega t / n)^{-n} N(x) & \text { for } x \in Y, \\
N(T(t ; n) x-x) \leqq t(1-|\omega| t / n)^{-n} N(A x) & \text { for } x \in Y_{1}, \\
T(t ; n) x-x=\int_{0}^{t} T(s ; n) \frac{n}{s} R\left(\frac{n}{s}\right) A x d s & \text { for } x \in Y_{1}, \\
R(\lambda) x-\int_{0}^{t} e^{-\lambda s} T(s ; n) x d s & \\
=e^{-\lambda t} T(t ; n) R(\lambda) x-\int_{0}^{t} e^{-\lambda s} T(s ; n) R\left(\frac{n}{s}\right) A R(\lambda) A x d s
\end{array}
$$

for $x \in Y_{1}$ and $\lambda>\omega$, where the integrals in (4.10) and (4.11) exist (with respect to the norm $N(\cdot)$ and a fortiori $\|\cdot\|)$.

Proof. (4.8) is an immediate consequence of Lemma 4.3. In view of the relation (4.6), $[\lambda R(\lambda)]^{n} x-x$ can be written as $\lambda^{-1} \sum_{k=1}^{n}[\lambda R(\lambda)]^{k} A x$ for $x \in Y_{1}$ and $\lambda>\omega$; hence by Lemma 4.3,

$$
N\left([\lambda R(\lambda)]^{n} x-x\right) \leqq \lambda^{-1} \sum_{k=1}^{n}\left(\frac{\lambda}{\lambda-|\omega|}\right)^{k} N(A x) \leqq \frac{n}{\lambda}\left(\frac{\lambda}{\lambda-|\omega|}\right)^{n} N(A x)
$$


for $x \in Y_{1}$ and $\lambda>|\omega|$. Taking $\lambda=n / t$, we obtain (4.9). Now, Lemma 4.3 and (4.1) assert that $R(\lambda)^{n} x, x \in Y$, are differentiable in $\lambda$ (with respect to the norm $N(\cdot)$ and $a$ fortiori $\|\cdot\|)$ and $(d / d \lambda) R(\lambda)^{n} x=-n R$ $(\lambda)^{n+1} x$ for $x \in Y, \lambda>\omega$ and $n \geqq 1$. Hence,

$$
T(t ; n) x-T(\varepsilon ; n) x=\int_{\varepsilon}^{t} T(s ; n) \frac{n}{s} R\left(\frac{n}{s}\right) A x d s, \quad 0<\varepsilon<t .
$$

Since $N\left(T(s ; n) \frac{n}{s} R\left(\frac{n}{s}\right) A x\right) \leqq(1-|\omega| t / n)^{-n-1} N(A x)$ for $0 \leqq s \leqq t$, the integrand is Bochner integrable on $(0, t)$. Also, $N(T(\varepsilon ; n) x-x) \rightarrow 0$ as $\varepsilon \rightarrow 0+$ by (4.9). Therefore, letting $\varepsilon \rightarrow 0+$, we have (4.10). Finally we prove (4.11). For $x \in Y_{1}$ and $\lambda>\omega$,

$$
\begin{aligned}
& (d / d s) e^{-\lambda s} T(s ; n) R(\lambda) x \\
& \quad=-\lambda e^{-\lambda s} T(s ; n) R(\lambda) x+e^{-\lambda s} T(s ; n) \frac{n}{s} R\left(\frac{n}{s}\right) A R(\lambda) x \\
& \quad=-e^{-\lambda s} T(s ; n) x+e^{-\lambda s} T(s ; n) R\left(\frac{n}{s}\right) A R(\lambda) A x
\end{aligned}
$$

Integrating both sides of this equality from $s=0$ to $s=t$, we get (4.11).

Q.E.D.

Now, we are in position to state the main result of this section.

Theorem 4.6. For each $x \in Y_{1}$, the $\|\cdot\|$-limit

$$
\lim _{n \rightarrow \infty} T(t ; n) x=\lim _{n \rightarrow \infty}\left(I-\frac{t}{n} A\right)^{-n} x
$$

exists uniformly on every bounded interval of $[0, \infty)$. If we define operators $T(t), t \geqq 0$, on $Y_{1}$ by

$$
T(t) x=\lim _{n \rightarrow \infty} T(t ; n) x
$$

for $t \geqq 0$ and $x \in Y_{1}$, then $\{T(t) ; t \geqq 0\}$ has the following properties:

(i) $\|T(t) x\| \leqq e^{\omega t} N(x) \quad$ for $x \in Y_{1}$ and $t \geqq 0$, 
(ii) $\|T(t) x-T(s) x\| \leqq e^{|\omega| \max (t, s)} N(A x)|t-s|$

for $s, t \geqq 0$ and $x \in Y_{1}$,

(iii) $T(t) x-x=\int_{0}^{t} T(s) A x d s \quad$ for $t \geqq 0$ and $x \in Y_{2}$,

(iv) $R(\lambda) x=\int_{0}^{\infty} e^{-\lambda t} T(t) x d t \quad$ for $\lambda>\omega$ and $x \in Y_{1}$,

(v) $N(x)=\sup _{t \geqq 0}\left\|e^{-\omega t} T(t) x\right\| \quad$ for $x \in Y_{1}$.

If in addition, $Y_{1}$ is $N(\cdot)$-dense in $Y$, then (4.12) is obtained for $x \in Y$ and the operators $T(t)$ are defined on $Y$ by (4.13); in this case, $\{T(t)$; $t \geqq 0\}$ satisfies (i), (iii), (iv) and (v) with $Y_{1}$ and $Y_{2}$ replaced by $Y$ and $Y_{1}$ respectively, and furthermore

(ii)' for each $x \in Y, T(t) x$ is $\|\cdot\|$-continuous in $t \geqq 0$.

Proof. It follows from Lemma 4.4 that for each $x \in Y_{1}$, the $\|\cdot\|$ limit (4.12) exists uniformly on every bounded sub-interval of $[0, \infty)$. (i) is an immediate consequence of (4.13) and (4.8). Next, by (4.10), we have

$$
\begin{aligned}
& \|T(t ; n) x-T(s ; n) x\| \leqq\left|\int_{s}^{t} N\left(T(\tau ; n) \frac{n}{\tau} R\left(\frac{n}{\tau}\right) A x\right) d \tau\right| \\
& \quad \leqq\left|\int_{s}^{t}(1-|\omega| \tau / n)^{-n-1} d \tau\right| N(A x) \leqq|t-s|(1-|\omega| \gamma / n)^{-n-1} N(A x)
\end{aligned}
$$

for $s, t \geqq 0, \gamma=\max \{t, s\}$ and $x \in Y_{1}$. (4.10) can be written as

$$
\begin{aligned}
T(t ; n) x-x & =\int_{0}^{t} T(s ; n) A x d s+\int_{0}^{t} T(s ; n)\left[\frac{n}{s} R\left(\frac{n}{s}\right) A x-A x\right] d s \\
& =\int_{0}^{t} T(s ; n) A x d s+\int_{0}^{t} T(s ; n) R\left(\frac{n}{s}\right) A^{2} x d s
\end{aligned}
$$

for $t \geqq 0$. Since

$$
N\left(T(s ; n) R\left(\frac{n}{s}\right) A^{2} x\right) \leqq \frac{s}{n}\left(1-|\omega| \frac{s}{n}\right)^{-n-1} N\left(A^{2} x\right)
$$




$$
\leqq \frac{t}{n}\left(1-|\omega| \frac{t}{n}\right)^{-n-1} N\left(A^{2} x\right)
$$

for $s \in(0, t]$, we have

$$
\left\|\int_{0}^{t} T(s ; n) R\left(\frac{n}{s}\right) A^{2} x d s\right\| \leqq \frac{t^{2}}{n}\left(1-|\omega| \frac{t}{n}\right)^{-n-1} N\left(A^{2} x\right) \rightarrow 0 \quad \text { as } n \rightarrow \infty,
$$

from which (iii) follows. Next, we prove (iv). Since

$$
N\left(T(s ; n) R\left(\frac{n}{s}\right) A R(\lambda) A x\right) \leqq \frac{t}{n}\left(1-|\omega| \frac{t}{n}\right)^{-n-1} N(A R(\lambda) A x)
$$

for $s \in(0, t]$ and $x \in Y_{1},(4.11)$ implies that

$$
\begin{aligned}
& \left\|R(\lambda) x-\int_{0}^{t} e^{-\lambda s} T(s ; n) x d s\right\| \\
& \leqq e^{-\lambda t}\left(1-\omega \frac{t}{n}\right)^{-n} N(R(\lambda) x)+e^{|\lambda| t} \frac{t^{2}}{n}\left(1-|\omega| \frac{t}{n}\right)^{-n-1} N(A R(\lambda) A x)
\end{aligned}
$$

for $x \in Y_{1}$ and $t \geqq 0$. Passing to the limit as $n \rightarrow \infty$, we have

$$
\left\|R(\lambda) x-\int_{0}^{t} e^{-\lambda s} T(s) x d s\right\| \leqq e^{-(\lambda-\omega) t} N(R(\lambda) x)
$$

for $x \in Y_{1}$ and $t \geqq 0$. Hence, (iv) is obtained by letting $t \rightarrow \infty$. Finally, we prove (v). The relation (4.1) and Lemma 4.3 imply that for each $x \in Y,(d / d \lambda)^{n-1} R(\lambda) x=(-1)^{n-1}(n-1) ! R(\lambda)^{n} x$ for $\lambda>\omega$ and $n \geqq 1$. On the other hand, we see using (iv) that

$$
(d / d \lambda)^{n-1} R(\lambda) x=(-1)^{n-1} \int_{0}^{\infty} e^{-\lambda t} t^{n-1} T(t) x d t
$$

for $x \in Y_{1}, \lambda>\omega$ and $n \geqq 1$, and hence we have

$$
R(\lambda)^{n} x=(n-1) !^{-1} \int_{0}^{\infty} e^{-\lambda t} t^{n-1} T(t) x d t, \quad x \in Y_{1}, \lambda>\omega, n \geqq 1 .
$$

Using this relation, we obtain

$$
\begin{aligned}
\left\|(\lambda-\omega)^{n} R(\lambda)^{n} x\right\| & \leqq(n-1) !^{-1}(\lambda-\omega)^{n} \int_{0}^{\infty} e^{-(\lambda-\omega) t} t^{n-1}\left\|e^{-\omega t} T(t) x\right\| d t \\
& \leqq \sup _{t \geqq 0}\left\|e^{-\omega t} T(t) x\right\|
\end{aligned}
$$


for $x \in Y_{1}, \lambda>\omega$ and $n \geqq 1$. This shows that $N(x) \leqq \sup _{t \geqq 0}\left\|e^{-\omega t} T(t) x\right\|$ for $x \in Y_{1}$. The opposite inequality is clear from (i). Now, assume that $Y_{1}$ is $N(\cdot)$-dense in $Y$. Then it follows from (4.8) that the convergence (4.12) holds uniformly on every bounded sub-interval of $[0, \infty)$ for all $x \in Y$. This fact states that (i) holds for $Y_{1}$ replaced by $Y$ and hence (ii) ${ }^{\prime}$ is obtained in view of (ii). To see that (iii) holds for all $x \in Y_{1}$, we first observe that for every $y \in Y$

$$
N(\lambda R(\lambda) y-y) \rightarrow 0 \quad \text { as } \lambda \rightarrow \infty \text {. }
$$

Indeed, if $y \in Y_{1}$ then $N(\lambda R(\lambda) y-y)=N(R(\lambda) A y) \leqq(\lambda-\omega)^{-1} N(A y) \rightarrow 0$ as $\lambda \rightarrow \infty$. From this and the $N(\cdot)$-denseness of $Y_{1}$ in $Y$, it follows that (4.15) holds for $y \in Y$. If $x \in Y_{1}$, then $\lambda R(\lambda) x \in Y_{2}$ and hence (iii) implies that

$$
T(t)[\lambda R(\lambda) x]-[\lambda R(\lambda) x]=\int_{0}^{t} T(s)[\lambda R(\lambda) A x] d s .
$$

Letting $\lambda \rightarrow \infty$ and using (4.15), we see that (iii) holds for all $x \in Y_{1}$. To show that (iv) holds for $Y_{1}$ replaced by $Y$, let $\lambda>\omega, x \in Y$ and choose $\left\{x_{n}\right\} \subset Y_{1}$ such that $N\left(x_{n}-x\right) \rightarrow 0$ as $n \rightarrow \infty$. Then by (iv), $R(\lambda) x_{n}=$ $\int_{0}^{\infty} e^{-\lambda t} T(t) x_{n} d t$ for each $n$. Hence, we have $R(\lambda) x=\int_{0}^{\infty} e^{-\lambda t} T(t) x d t$, because of that $\left\|R(\lambda) x_{n}-R(\lambda) x\right\| \leqq(\lambda-\omega)^{-1} N\left(x_{n}-x\right) \rightarrow 0$ and also of that $\left\|T(t) x_{n}-T(t) x\right\| \leqq e^{\omega t} N\left(x_{n}-x\right) \rightarrow 0$ as $n \rightarrow \infty$. Finally, if $Y_{1}$ is $N(\cdot)$. dense in $Y$, then (4.14) holds for every $x \in Y, \lambda>\omega$ and $n \geqq 1$; hence (v) is obtained for $Y_{1}$ replaced by $Y$.

Q.E.D.

\section{Construction of Semi-Groups}

Let $A$ be a linear operator in a Banach space $X$ and $Y$ be a linear subset of $X$ satisfying conditions $\left(a_{1}\right)-\left(a_{3}\right)$ which have been introduced in the preceding section. We then define a linear subset $\widetilde{\Sigma}$ of $X$ and a function $\tilde{N}(\cdot)$ on $\widetilde{\Sigma}$ by

$$
\widetilde{\Sigma}=\left\{x \in \bigcap_{\lambda>\omega} \bigcap_{n \geqq 0} D\left(R(\lambda)^{n}\right) ; \sup _{n \geqq 0}, \lambda>\omega\left\|(\lambda-\omega)^{n} R(\lambda)^{n} x\right\|<\infty\right\},
$$




$$
\tilde{N}(x)=\sup _{n \geqq 0, \lambda>\omega}\left\|(\lambda-\omega)^{n} R(\lambda)^{n} x\right\| \quad \text { for } x \in \widetilde{\Sigma} .
$$

Obviously, $\|x\| \leqq \tilde{N}(x)$ for $x \in \widetilde{\Sigma}$. Also, the function $\tilde{N}(\cdot)$ defines a norm on $\widetilde{\Sigma}$ and

$$
Y \subset \widetilde{\Sigma} \tilde{N}(x)=N(x)(\leqq M\|x\|) \quad \text { for } x \in Y .
$$

In this section we first construct a semi-group of class $\left(C_{0}\right)$ on a certain Banach space which is contained in the above-mentioned $\widetilde{\Sigma}$ as its linear subset and then discuss to extend this semi-group to a semi-group on $X$, by imposing a condition of Feller type. The results obtained will be applied to establish the generation theorems of the semi-groups which we discussed in Sections 2 and 3.

5.1. Throughout this subsection, let $A$ be a linear operator in $X$ and $Y$ be a linear subset of $X$ satisfying $\left(\mathrm{a}_{1}\right)-\left(\mathrm{a}_{3}\right)$ and the following conditions:

(a) $\quad Y_{1}$ is $N(\cdot)$-dense in $Y$,

(B) $\widetilde{\Sigma}$ is a Banach space under the norm $\tilde{N}(\cdot)$.

We note that (B) is satisfied if $A$ is closed in $X$, as will be shown in Lemma 5.3.

Now, let us denote by $\tilde{Y}$ the $\tilde{N}(\cdot)$-closure of $Y$. Then, under condition (B), $\tilde{Y}$ becomes a Banach space under $\tilde{N}(\cdot)$. We shall construct a semi-group of class $\left(C_{0}\right)$ on this Banach space. First, Lemma 4.3 and (5.3) state that $\tilde{N}((\lambda-\omega) R(\lambda) x) \leqq \tilde{N}(x)$ for $x \in Y$ and $\lambda>\omega$. Hence, for each $\lambda>\omega, R(\lambda) \mid Y$ has a unique extension $\tilde{R}(\lambda)$ onto $\tilde{Y}$. $\tilde{R}(\lambda)$ maps $\tilde{Y}$ into itself and

$$
\tilde{N}((\lambda-\omega) \tilde{R}(\lambda) x) \leqq \tilde{N}(x) \quad \text { for } x \in \tilde{Y} \text { and } \lambda>\omega .
$$

In view of this fact, for each $t \geqq 0$ and each positive integer $n>\omega t$, we can define a linear operator $\tilde{T}(t ; n)$ from $\tilde{Y}$ into itself, by

$$
\left.\widetilde{T}(t ; n) x=\left\{\frac{n}{t} \tilde{R}\left(\frac{n}{t}\right)\right\}^{n} x \text { (if } t>0\right),=x \text { (if } t=0 \text { ), for } x \in \tilde{Y} \text {. }
$$


Clearly, $T(t ; n) x=T(t ; n) x$ for $x \in Y$ and

$$
\tilde{N}(\tilde{T}(t ; n) x) \leqq(1-\omega t / n)^{-n} \tilde{N}(x) \quad \text { for } x \in \tilde{Y}, t \geqq 0 \text { and } n>\omega t
$$

Since $Y_{1}$ is $\tilde{N}(\cdot)$-dense in $\tilde{Y}$, Lemma 4.4 and (5.6) imply that for each $x \in \tilde{Y}$,

$$
\widetilde{T}(t) x \equiv \tilde{N}(\cdot)-\lim _{n \rightarrow \infty} \tilde{T}(t ; n) x
$$

holds uniformly for $t$ in every bounded sub-interval of $[0, \infty)$. As is easily seen, $\tilde{T}(t) \in B(\tilde{Y})$ for $t \geqq 0$ and

$$
\tilde{N}(\tilde{T}(t) x) \leqq e^{\omega t} \tilde{N}(x) \quad \text { for } x \in \tilde{Y} \text { and } t \geqq 0
$$

Theorem 5.1. $\{\tilde{T}(t) ; t \geqq 0\}$ forms a semi-group of class $\left(C_{0}\right)$ on $\tilde{Y}$ such that $\tilde{N}(\tilde{T}(t) x) \leqq e^{\omega t} \tilde{N}(x)$ for $x \in \tilde{Y}$ and $t \geqq 0$, and its infinitesimal generator is the $\tilde{N}(\cdot)$-closure $\overparen{A \mid Y_{1}}$ of $A \mid Y_{1}$. Moreover, $\tilde{R}(\lambda)=$ $\left(\lambda-\widetilde{A \mid Y_{1}}\right)^{-1}$ for $\lambda>\omega$ and $\tilde{T}(t) \mid Y=T(t)$ for $t \geqq 0$, where $T(t), t \geqq 0$, are the operators defined by (4.13).

Proof. Since $\widetilde{T}(t ; n)|Y=T(t ; n)| Y$, Lemma 4.5 yields that $\widetilde{T}(t ; n) x$ is $\tilde{N}(\cdot)$-continuous in $t \in[0, \beta]$ for $x \in Y_{1}, n>|\omega| \beta$, and $\beta>0$. Hence, $\tilde{T}(t) x$ is $\tilde{N}(\cdot)$-continuous in $t \geqq 0$ for each $x \in Y_{1}$. This also remains true for all $x \in \tilde{Y}$ by (5.8) and condition $\left(\mathrm{a}_{4}\right)$. Also, we can prove the semi-group property $\widetilde{T}(t+s)=\widetilde{T}(t) \widetilde{T}(s), t, s \geqq 0$, by the usual method. Consequently, $\{\widetilde{T}(t) ; t \geqq 0\}$ forms a semi-group of class $\left(C_{0}\right)$ on $\tilde{Y}$. It is obvious that $\widetilde{T}(t)|Y=T(t)| Y$ for $t \geqq 0$; hence by theorem 4.6, we have

$$
\begin{gathered}
\widetilde{T}(t) x-x=\int_{0}^{t} \tilde{T}(s) A x d s \quad \text { for } x \in Y_{1} \text { and } t \geqq 0, \\
\tilde{R}(\lambda) x(=R(\lambda) x)=\int_{0}^{\infty} e^{-\lambda t} \tilde{T}(t) x d t \quad \text { for } x \in Y \text { and } \lambda>\omega,
\end{gathered}
$$

the integrals being taken with respect to the norm $\tilde{N}(\cdot)$. Let $B$ be the infinitesimal generator of the semi-group $\{\widetilde{T}(t) ; t \geqq 0\}$ on $\tilde{Y}$. In view of (5.8), we see that $B$ is $\tilde{N}(\cdot)$-closed and 


$$
(\lambda-B)^{-1} x=\int_{0}^{\infty} e^{-\lambda t} \widetilde{T}(t) x d t, \quad \text { for } \lambda>\omega \text { and } x \in \tilde{Y}
$$

Combining this with (5.10), $\tilde{R}(\lambda) x=\int_{0}^{\infty} e^{-\lambda t} \widetilde{T}(t) x d t$ for $\lambda>\omega$ and $x \in \tilde{Y}$; hence $\tilde{R}(\lambda)=(\lambda-B)^{-1}$ for $\lambda>\omega$. Also, we see from (5.9) that $\overparen{A \mid Y_{1}} \subset$ $B$. We further demonstrate that $\overparen{A \mid Y_{1}}=B$. Let $x \in D(A)$. Then there is an element $y \in \tilde{Y}$ such that $x=\widetilde{R}(\lambda) y\left(=(\lambda-B)^{-1} y\right)$. Choose $\left\{y_{n}\right\} \subset$ $Y_{1}$ so that $\tilde{N}\left(y_{n}-y\right) \rightarrow 0$ as $n \rightarrow \infty$ and set $x_{n}=\tilde{R}(\lambda) y_{n}\left(=R(\lambda) y_{n}\right), n \geqq 1$. Then, $x_{n} \in Y_{2}\left(\subset Y_{1}\right)$ and $\tilde{N}\left(x_{n}-x\right) \leqq(\lambda-\omega)^{-1} \tilde{N}\left(y_{n}-y\right) \rightarrow 0$ as $n \rightarrow \infty$. Moreover, $\tilde{N}\left(\left(A \mid Y_{1}\right) x_{n}-B x\right)=\tilde{N}\left(A R(\lambda) y_{n}-B \tilde{R}(\lambda) y\right)=\tilde{N}\left(\left(\lambda R(\lambda) y_{n}-y_{n}\right)-\right.$ $(\lambda \tilde{R}(\lambda) y-y)) \leqq \lambda \tilde{N}\left(x_{n}-x\right)+\tilde{N}\left(y_{n}-y\right) \rightarrow 0$ as $n \rightarrow \infty$. This means that $\widetilde{A \mid Y_{1}}=B$. Thus, $\tilde{R}(\lambda)=\left(\lambda-\overparen{A \mid Y_{1}}\right)^{-1}$ for $\lambda>\omega$.

Q.E.D.

Remark 5.2. If we do not assume condition $\left(\mathrm{a}_{4}\right)$, then for each $x \in$ $\tilde{Y}_{1}, \widetilde{T}(t) x \equiv \tilde{N}(\cdot)-\lim _{n \rightarrow \infty} \tilde{T}(t ; n) x$ exists uniformly on every bounded subinterval of $[0, \infty)$, where $\tilde{Y}_{1}$ is the $\tilde{N}(\cdot)$-closure of $Y_{1} . \quad\{\tilde{T}(t) ; t \geqq 0\}$ becomes a semi-group of class $\left(C_{0}\right)$ on $\tilde{Y}_{1}$ such that $\tilde{N}(\tilde{T}(t) x) \leqq e^{\omega t} \tilde{N}(x)$ for $x \in \tilde{Y}_{1}$ and $t \geqq 0$; its infinitesimal generator is $\overparen{A \mid Y_{2}}$. Moreover, $\widetilde{T}(t)\left|Y_{1}=T(t)\right| Y_{1}$ for $t \geqq 0$, where $T(t), t \geqq 0$, are the operators defined by (4.13).

5.2. Throughout this subsection, we assume condition $\left(a_{4}\right)$ which has been imposed in the preceding subsection and furthermore that $A$ is closed in $X$. In this case, we do not need to suppose condition (B), see Feller [3; Theorem 1]:

Lemma 5.3. The space $\widetilde{\Sigma}$ becomes a Banach space under $\tilde{N}(\bullet)$.

By means of this lemma, all conclusions obtained in Section 5.1 hold under the assumptions in this subsection. Also, we see from the closedness of $A$ and $R(\lambda)$ that

$$
\begin{aligned}
& \widetilde{A \mid Y_{1}} \subset A \text { and } \tilde{R}(\lambda) \subset R(\lambda) \quad \text { for } \lambda>\omega \text {, } \\
& \widetilde{T}(t ; n) x=\left\{\frac{n}{t} R\left(\frac{n}{t}\right)\right\}^{n} x=\left(I-\frac{t}{n} A\right)^{-n} x
\end{aligned}
$$


for $x \in \tilde{Y}$ and $t>0$. Combining these with Theorem 5.1, we obtain

Theorem 5.4. For each $x \in \tilde{Y}$,

$$
\widetilde{T}(t) x \equiv \hat{N}(\cdot)-\lim _{n \rightarrow \infty}\left(I-\frac{t}{n} A\right)^{-n} x
$$

exists uniformly on every bounded subinterval of $[0, \infty)$. The family $\{\widetilde{T}(t)$; $t \geqq 0\}$ forms a semi-group of class $\left(C_{0}\right)$ on $\tilde{Y}$ such that $\tilde{N}(\widetilde{T}(t) x) \leqq$ $e^{\omega t} \tilde{N}(x)$ for $x \in \tilde{Y}$ and $t \geqq 0$. Its infinitesimal generator is $A \mid R(\lambda)[\tilde{Y}]$ and $R(\lambda) \mid \tilde{Y}, \lambda>\omega$, are the resolvents of the infinitesimal generator. Moreover, $\widetilde{T}(t) x=T(t) x$ for $t \geqq 0$ and $x \in Y$, where $T(t), t \geqq 0$, are the operators defined by (4.13).

Proof. By theorem 5.1, $\widetilde{A \mid Y_{1}}$ is the infinitesimal generator of the semi-group $\{\widetilde{T}(t) ; t \geqq 0\}$ and $\tilde{R}(\lambda)$ is the resolvent of ${\widetilde{A \mid Y_{1}}}_{1}$ at $\lambda(>\omega)$. But, $\tilde{R}(\lambda)=R(\lambda) \mid \tilde{Y}$ by $(5.11)$, and so, $D\left(\widetilde{A \mid Y_{1}}\right)=\tilde{R}(\lambda)[\tilde{Y}]=R(\lambda)[\tilde{Y}]$. Since $\overparen{A \mid Y_{1}} \subset A$, it follows that $A \mid Y_{1}=\widetilde{A \mid R}(\lambda)[\tilde{Y}]$ Q.E.D.

Remark 5.5. If we do not assume condition $\left(\mathrm{a}_{4}\right)$, then for each $x \in \tilde{Y}_{1}$, $\tilde{T}(t) x \equiv \tilde{N}(\cdot)-\lim _{n \rightarrow \infty}\left(I-\frac{t}{n} A\right)^{-n} x$ exists uniformly on every bounded subinterval of $[0, \infty)$. The family $\left\{e^{-\omega t} \widetilde{T}(t) ; t \geqq 0\right\}$ forms a contraction semi-group of class $\left(C_{0}\right)$ on $\tilde{Y}_{1}$ whose infinitesimal generator is $\overparen{A \mid Y_{2}}=$ $A\left|R(\lambda)\left[\tilde{Y}_{1}\right] ; R(\lambda)\right| \tilde{Y}_{1}$ is the resolvent of $\widetilde{A \mid Y_{2}}$ at $\lambda(>\omega)$ and $\tilde{T}(t) \mid Y_{1}$ $=T(t) \mid Y_{1}$ for $t \geqq 0$.

5.3. Let $(A, Y)$ be a pair of linear operator and linear manifold in $X$ satisfying $\left(\mathrm{a}_{1}\right)-\left(\mathrm{a}_{4}\right)$ and $(\mathrm{B})$ and let $\{\widetilde{T}(t) ; t \geqq 0\}$ be a semi-group on $\tilde{Y}$ obtained by Theorem 5.1. $\widetilde{T}(t)$ need not be bounded on the linear subset $Y$ of $X$ if it is observed in the original space $X$. In this subsection we extend this semi-group $\{\widetilde{T}(t) ; t \geqq 0\}$ on $\tilde{Y}$ to a semi-group on $X$ by imposing the following two conditions:

(a) $Y$ is dense in $X$,

$\left(\mathrm{a}_{6}\right)$ for every $\varepsilon>0$ and $x \in Y$ there exist $M_{\varepsilon}>0$ and $\lambda_{0}=\lambda_{0}(\varepsilon, x)$ such that for $\lambda>\lambda_{0}$ and $n$ with $n / \lambda \in[\varepsilon, 1 / \varepsilon]$, 


$$
\left\|[\lambda R(\lambda)]^{n} x\right\| \leqq M_{\varepsilon}\|x\| .
$$

Let $T(t, n)$ and $T(t)$ be the operators on $Y$ defined by (4.7) and (4.13) for the case in which $Y_{1}$ is $N(\cdot)$-dense in $Y$, respectively. Condition $\left(\mathrm{a}_{6}\right)$ states that for each $x \in Y$,

$$
\|T(t ; n) x\| \leqq M_{\varepsilon}\|x\| \quad \text { for } t \in[\varepsilon, 1 / \varepsilon] \text { and } n \text { sufficiently large. }
$$

Therefore $T(t) x$ satisfies

$$
\|T(t) x\| \leqq M_{\varepsilon}\|x\| \quad \text { for } t \in[\varepsilon, 1 / \varepsilon] \text { and } x \in Y \text {. }
$$

This means that for each $t>0, T(t)$ is a bounded linear operator on $Y$ as an operator in $X$. According to $\left(\mathrm{a}_{5}\right)$, each $T(t)$ can be uniquely extended to an element of $B(X)$. We denote this extension again by the same symbol $T(t)$. Then we have

$$
\|T(t) x\| \leqq M_{\varepsilon}\|x\| \quad \text { for } t \in[\varepsilon, 1 / \varepsilon] \text { and } x \in X \text {. }
$$

Now, setting $T(0)=I$, we demonstrate that $\{T(t) ; t \geqq 0\}$ forms a semi-group on $X$. In fact, by Theorem 5.1, $T(t)|Y=\tilde{T}(t)| Y$ for $t \geqq 0$ and $\{\widetilde{T}(t) ; t \geqq 0\}$ is a semi-group of class $\left(C_{0}\right)$ on the Banach space $\tilde{Y}$. Hence, $T(t) \mid \tilde{Y}=\widetilde{T}(t)$ for $t \geqq 0$, and so, $T(t+s) x=\widetilde{T}(t+s) x=\widetilde{T}(t) \widetilde{T}(s) x$ $=T(t) T(s) x$ for $t, s \geqq 0$ and $x \in Y$. Since $\tilde{Y}$ is dense in $X$ by $\left(\mathrm{a}_{5}\right)$, we obtain the semi-group property $T(t+s)=T(t) T(s)$ for $t, s \geqq 0$. Also, since $T(t) x$ is continuous in $t \geqq 0$ for each $x \in \tilde{Y}$ and since $\tilde{Y}$ is dense in $X,(5.16)$ implies that $T(t) x$ is continuous in $t>0$ for each $x \in X$. Thus, $\{T(t) ; t \geqq 0\}$ forms a semi-group on $X$. Furthermore, $A \mid Y_{1}$ is a restriction of the infinitesimal generator of $\{T(t) ; t \geqq 0\}$. Indeed, since $\widetilde{A \mid Y_{1}}$ is the infinitesimal generator of $\{\widetilde{T}(t) ; t \geqq 0\}$, if $x \in Y_{1}$ then

$$
\left(A \mid Y_{1}\right) x=\left(\widetilde{A \mid Y_{1}}\right) x=\lim _{h \rightarrow 0+} h^{-1}(\widetilde{T}(h) x-x)=\lim _{h \rightarrow 0^{+}} h^{-1}(T(h) x-x) .
$$

We can summarize the above-mentioned as follows:

Theorem 5.6. Assume conditions $\left(\mathrm{a}_{1}\right)-\left(\mathrm{a}_{6}\right)$ and $(\mathrm{B})$. Then there is a semi-group $\{T(t) ; t \geqq 0\}$ on $X$ such that 


$$
T(t) x=\lim _{n \rightarrow \infty}\left(I-\frac{t}{n} A\right)^{-n} x \quad \text { for } x \in Y \text { and } t \geqq 0 \text {, }
$$

where the convergence is uniform with respect to $t$ in every bounded subinterval of $[0, \infty)$. Moreover,

(i) for each $x \in Y, T(t) x$ is continuous in $t \geqq 0$,

(ii) $A \mid Y_{1}$ is a restriction of the infinitesimal generator,

(iii) $R(\lambda) x=\int_{0}^{\infty} e^{-\lambda t} T(t) x d t \quad$ for $x \in Y$ and $\lambda>\omega$,

(vi) $\quad N(x)=\sup _{t \geqq 0}\left\|e^{-\omega t} T(t) x\right\| \quad$ for $x \in Y$ (cf. Theorem 4.6).

Remark 5.7. Let us consider the following $\left(\mathrm{a}_{6}{ }^{\prime}\right)$, instead of $\left(\mathrm{a}_{6}\right)$ :

$\left(\mathrm{a}_{6}{ }^{\prime}\right)$ for every $\varepsilon>0$ and $x \in Y$ there exist $K_{\varepsilon}>0$ and $\lambda_{0}=\lambda_{0}(\varepsilon, x)$ such that for $\lambda>\lambda_{0}$ and $n$ with $n \geqq(\lambda-\omega) \varepsilon$,

$$
\left\|(\lambda-\omega)^{n} R(\lambda)^{n} x\right\| \leqq K_{\varepsilon}\|x\| \text {. }
$$

We observe that $\left(a_{6}{ }^{\prime}\right)$ implies $\left(a_{6}\right)$. See Remark 2.5. If $\left(a_{6}\right)$ is replaced by $\left(\mathrm{a}_{6}{ }^{\prime}\right)$, then the semi-group $\{T(t) ; t \geqq 0\}$ on $X$ has the property that for every $\varepsilon>0,\left\|e^{-\omega t} T(t)\right\|$ is bounded on $[\varepsilon, \infty)$. Indeed, if $t \geqq \varepsilon>0$ and $x \in Y$, then a constant $M_{\varepsilon}>0$ can be found such that $\|T(t ; n) x\|=(n /$ $(n-\omega t))^{n}\left\|(n / t-\omega)^{n} R\left(\frac{n}{t}\right)^{n} x\right\| \leqq M_{\varepsilon}(1-\omega t / n)^{-n}\|x\|$ for all sufficiently large $n$. Now, we see taking the limit as $n \rightarrow \infty$ that $\|T(t) x\| \leqq M_{\varepsilon} e^{\omega t}$ $\|x\|$ for $t \geqq \varepsilon$ and $x \in Y$. Since $\bar{Y}=X$, we have the desired conclusion.

\section{Generation Theorems}

In this section we give some necessary and sufficient conditions for given operators to be the infinitesimal generators of the semi-groups which we discussed in Sections 2 and 3.

We start from the following

Theorem 6.1. Let $Y$ be a dense linear subset of a Banach space $X$ 
and let $A$ be a linear operator with domain and range in $Y$. Assume that

$\left(\mathrm{c}_{1}\right)$ there is a real $\omega$ such that for each $\lambda>\omega$ and $x \in Y$, the equation $(\lambda-A) y=x$ has a unique solution $y=R(\lambda) x$,

(c $\left.c_{2}\right) \quad N(x)=\sup _{n \geqq 0, \lambda>\omega}\left\|(\lambda-\omega)^{n} R(\lambda)^{n} x\right\|<\infty$ for every $x \in Y$ and $Y$ is a Banach space under the norm $N(\cdot)$,

$\left(\mathrm{c}_{3}\right) D(A)$ is $N(\cdot)$-dense in the Banach space $Y$,

$\left(c_{4}\right)$ for every $\varepsilon>0$ and $x \in Y$ there exist $K_{\varepsilon}>0$ and $\lambda_{0}=\lambda_{0}(\varepsilon, x) \in R$ such that for $\lambda>\lambda_{0}$ and $n$ with $n \geqq(\lambda-\omega) \varepsilon$,

$$
\left\|(\lambda-\omega)^{n} R(\lambda)^{n} x\right\|_{1} \leqq K_{\varepsilon}\|x\| .
$$

Then the operator $A$ is the infinitesimal generator in the sense of Feller of a semi-group $\{T(t) ; t \geqq 0\}$ on $X$ such that $X_{0} \equiv \bigcup_{t>0} T(t)[X]$ is dense in $X$ and such that $Y$ is the continuity set of this semi-group. Moreover, $\{T(t) ; t \geqq 0\}$ is determined by

$$
T(t) x=\lim _{n \rightarrow \infty}\left(I-\frac{t}{n} A\right)^{-n} x \quad \text { for } x \in Y \text { and } t \geqq 0
$$

where the convergence is uniform with respect to $t$ in every bounded subinterval of $[0, \infty)$ for each $x \in Y$.

Proof. Noting that $D(A) \subset Y$ and $R(A) \subset Y$, we see that $\left(\mathrm{c}_{1}\right)$ and ( $\left.\mathrm{c}_{2}\right)$ imply conditions $\left(\mathrm{a}_{1}\right)-\left(\mathrm{a}_{3}\right)$ with $\|x\|=N(x)$ and the range condition $R(\lambda-A)=Y$ for $\lambda>\omega$. Furthermore, $Y_{1}=D(A)$ and $Y=\tilde{Y}=\widetilde{\Sigma}$ and hence $N(\cdot)=\tilde{N}(\cdot)$. Therefore $\left(\mathrm{c}_{2}\right)$ and $\left(\mathrm{c}_{3}\right)$ imply conditions $(\mathrm{B})$ and $\left(\mathrm{a}_{4}\right)$, respectively. $\left(c_{4}\right)$ is nothing but $\left(a_{6}{ }^{\prime}\right)$. Thus, it follows from Theorem 5.6 and Remark 5.7 that there exists a semi-group $\{T(t) ; t \geqq 0\}$ on $X$ determined by (6.1), such that $Y \subset \sum$ and $A \subset A_{0}$, where $\Sigma$ denotes the continuity set of $\{T(t) ; t \geqq 0\}$ and where $A_{0}$ stands for the infinitesimal generator. Moreover, we have the following relations:

$$
\begin{array}{ll}
N(x)=\sup _{t \geqq 0}\left\|e^{-\omega t} T(t) x\right\| & \text { for } x \in Y, \\
R(\lambda) x=\int_{0}^{\infty} e^{-\lambda t} T(t) x d t \quad \text { for } x \in Y \text { and } \lambda>\omega,
\end{array}
$$



for every $\varepsilon>0$ there is an $M_{\varepsilon}>0$ such that $\left\|e^{-\omega t} T(t)\right\| \leqq M_{\varepsilon}$ for $t \geqq \varepsilon$.

To show that $Y \supset \sum$, we first prove that

$$
X_{0} \equiv \bigcup_{t>0} T(t)[X] \subset Y .
$$

Let $x \in X$ and $t>0$. Since $\bar{Y}=X$, a sequence $\left\{x_{n}\right\} \subset Y$ can be found such that $\left\|x_{n}-x\right\| \rightarrow 0$ as $n \rightarrow \infty$. Since $\widetilde{T}(t): \tilde{Y} \rightarrow \tilde{Y}, \tilde{Y}=Y$, and since $\widetilde{T}(t)=T(t) \mid Y$, we have that $T(t)[Y] \subset Y$; hence $T(t) x_{n} \in Y$. Now, (6.2) and (6.4) yield that $N\left(T(t) x_{n}-T(t) x_{m}\right) \leqq M_{t} e^{\omega t}\left\|x_{n}-x_{m}\right\| \rightarrow 0$ as $m, n \rightarrow$ $\infty$. Thus, there is an element $y \in Y$ such that $N\left(T(t) x_{n}-y\right) \rightarrow 0$ and a fortiori $\left\|T(t) x_{n}-y\right\| \rightarrow 0$ as $n \rightarrow \infty$. But, $\left\|T(t) x_{n}-T(t) x\right\| \rightarrow 0$ as $n \rightarrow$ $\infty$, and so, $T(t) x=y \in Y$. Let $x \in \sum$. Then $T(t) x$ is uniformly $\|\cdot\|$. continuous on every bounded sub-interval of $[0, \infty)$. Combining this with (6.4), we obtain $N\left(T(h) x-T\left(h^{\prime}\right) x\right)=\sup _{s \geq 0}\left\|e^{-\omega s}\left(T(s+h) x-T\left(s+h^{\prime}\right) x\right)\right\|$ $\rightarrow 0$ as $h, h^{\prime} \rightarrow 0+$, note that $T(h) x \in Y$ for $h>0$ by (6.5). Thus, there exists a $y \in Y$ such that $\|T(h) x-y\| \leqq N(T(h) x-y) \rightarrow 0$ as $h \rightarrow 0+$. On the other hand, $\|T(h) x-x\| \rightarrow 0$ as $h \rightarrow 0+$, and so, $x=y \in Y$. This shows that $\Sigma \subset Y$. Therefore we have $Y=\Sigma$. The range $X_{0}$ of the semi-group $\{T(t) ; t \geqq 0\}$ is dense in $X$ since it is dense in $\Sigma=Y$. Finally, we prove that $A$ coincides with $\Omega$, the infinitesimal generator in the sense of Feller of $\{T(t) ; t \geqq 0\}$. Since $A \subset A_{0}$,

$$
\lim _{h \rightarrow 0+} h^{-1}[T(h) x-x]=A x \in Y \quad \text { for } x \in D(A),
$$

so that $A \subset \Omega$. Let $y \in D(\Omega)$. Then, by Corollary 2.3 (i), there is an $x \in \Sigma$ such that $y=J(\lambda) x=\int_{0}^{\infty} e^{-\lambda t} T(t) x d t$. Since $x \in Y(=\Sigma)$, (6.3) states that $y=\int_{0}^{\infty} e^{-\lambda t} T(t) x d t=R(\lambda) x \in D(A)$. This means that $D(\Omega) C$ $D(A)$. Consequently, $A=\Omega$.

Q.E.D.

Remark. The above theorem is the converse of Theorem 2.6. This theorem is proved for the case $\omega=0$ in Feller [3]. In [3] it is assumed, together with $\left(\mathrm{c}_{1}\right) \cdot\left(\mathrm{c}_{4}\right)$, that $N(\lambda R(\lambda) x) \leqq N(x)$ for $x \in Y$ and $\lambda>0$. But, this assumption is superfluous, as is seen from our proof, 
Theorem 6.2. Let $Y$ be a linear subset of a Banach space $X$ and let $A$ be a densely defined, closed linear operator in $X$. Assume that

$\left(\mathrm{a}_{1}\right) \quad Y$ is a normed space under a certain norm $\|\cdot\| \|$,

$\left(\mathrm{a}_{2}\right)$ there is a real $\omega$ such that for each $\lambda>\omega, R(\lambda-A) \supset Y, R(\lambda) \equiv$ $(\lambda-A)^{-1}$ exists and $R(\lambda)[Y] \subset Y$,

$\left(\mathrm{a}_{3}\right)$ there exists a constant $M>0$ such that

$$
\left\|R(\lambda)^{n} x\right\| \leqq M(\lambda-\omega)^{-n}\|x\| \quad \text { for } x \in Y, \lambda>\omega \text { and } n \geqq 0,
$$

(a) $Y_{1}=\{x \in Y ; A x \in Y\}$ is $N(\cdot)$-dense in $Y$, where $N(\cdot)$ is defined by $N(x)=\sup _{n \geq 0, \lambda>\omega}\left\|(\lambda-\omega)^{n} R(\lambda)^{n} x\right\|$ for $x \in Y$,

$\left(a_{6}\right)$ for every $\varepsilon>0$ and $x \in Y$, there exist $M_{\varepsilon}>0$ and $\lambda_{0}=\lambda_{0}(\varepsilon, x)$ such that

$$
\left\|\lambda^{n} R(\lambda)^{n} x\right\| \leqq M_{\varepsilon}\|x\| \quad \text { for } \lambda>\lambda_{0} \text { and } n \text { with } n / \lambda \in[\varepsilon, 1 / \varepsilon]
$$

(a) $Y_{1}$ is a core of $A$.

Then $A$ is the complete infinitesimal generator of a semi-group $\{T(t)$; $t \geqq 0\}$ on $X$ such that $X_{0} \equiv \bigcup_{t>0} T(t)[X]$ is dense in $X$ and such that $Y$ is contained in the continuity set $\sum$ of this semi-group. Moreover, this semi-group is determined by (6.1).

Proof. $\quad\left(\mathrm{a}_{7}\right)$ and the denseness of $D(A)$ imply $\left(\mathrm{a}_{5}\right)$, i.e., $\bar{Y}=X$. The closedness of $A$ implies (B) by Lemma 5.3. Hence, by Theorem 5.6, there is a semi-group $\{T(t) ; t \geqq 0\}$ on $X$ determined by (6.1) such that $Y \subset \Sigma$ and such that $A \mid Y_{1} \subset A_{0}$, where $A_{0}$ is the infinitesimal generator of $\{T(t) ; t \geqq 0\}$. Recall (Theorem 5.4) that $R(\lambda) \mid \tilde{Y}, \lambda>\omega$, are the resolvents of the infinitesimal generator of the semi-group $\{\widetilde{T}(t) ; t \geqq 0\}$ of class $\left(C_{0}\right)$ on the Banach space $\tilde{Y}$. Therefore $R(\lambda) \mid \tilde{Y}$ can be expressed by $R(\lambda) x=\int_{0}^{\infty} e^{-\lambda t} \widetilde{T}(t) x d t$ for $x \in \tilde{Y}$ and $\lambda>\omega$. Since $T(t) \mid \tilde{Y}=\widetilde{T}(t)$ for $t \geqq 0$ (see Section 5.3), we see that $T(t)[\tilde{Y}] \subset \tilde{Y}$ for $t \geqq 0$ and

$$
R(\lambda) x=\int_{0}^{\infty} e^{-\lambda t} T(t) x d t \quad \text { for } x \in \tilde{Y} \text { and } \lambda>\omega
$$

Let $\omega_{0}$ be the type of $\{T(t) ; t \geqq 0\}$ on $X$ and let $\omega_{1}>\omega_{0}$. For each $s>0$ there is an $M(s)>0$ such that $\|T(t)\| \leqq M(s) \exp \left(\omega_{1} t\right)$ for $t \geqq s$. We 
want to show that $T(s) x \in D(R(\lambda))$ and

$$
R(\lambda) T(s) x=\int_{0}^{\infty} e^{-\lambda t} T(t+s) x d t
$$

for $x \in X, s>0$ and $\lambda>\max \left\{\omega_{1}, \omega\right\}$. Since $\bar{Y}=X$, one can choose a sequence $\left\{x_{n}\right\} \subset Y$ such that $\left\|x_{n}-x\right\| \rightarrow 0$ as $n \rightarrow \infty$. Since $T(s) x_{n} \in \tilde{Y}$, it follows from (6.6) that

$$
R(\lambda) T(s) x_{n}=\int_{0}^{\infty} e^{-\lambda t} T(t+s) x_{n} d t
$$

Since $\left\|e^{-\lambda t} T(t+s) x_{n}\right\| \leqq M(s) \exp \left(\omega_{1} s\right)\left\|x_{n}\right\| \exp \left(-\left(\lambda-\omega_{1}\right) t\right) \leqq M^{\prime}(s) \exp (-$ $\left.\left(\lambda-\omega_{1}\right) t\right) \in L(0, \infty)$, we see applying the dominated convergence theorem that $R(\lambda) T(s) x_{n}=\int_{0}^{\infty} e^{-\lambda t} T(t+s) x_{n} d t \rightarrow \int_{0}^{\infty} e^{-\lambda t} T(t+s) x d t$ as $n \rightarrow \infty$. Additionally, $\lim _{n \rightarrow \infty} T(s) x_{n}=T(s) x$. Thus, (6.7) is obtained from the closedness of $R(\lambda)$. Now, (6.7) states that $R_{0}(\lambda) \subset R(\lambda)$ for $\lambda>\max \left\{\omega_{1}, \omega\right\}$ ( $\left.>\omega_{0}\right), R_{0}(\lambda)$ being the operator on $X_{0}$ defined by (3.1). Since $R(\lambda)$ is closed and invertible, $R_{0}(\lambda)$ is closable and its closure $\overline{R_{0}(\lambda)}$ is invertible for each $\lambda>\max \left\{\omega_{1}, \omega\right\}$; hence condition $\left(\alpha_{1}\right)$ introduced in Section 3 is satisfied. Therefore, by Lemma 3.1, $\bar{A}_{0}=\lambda-\bar{R}_{0}(\lambda)^{-1} \subset \lambda-R(\lambda)^{-1}=A$. Combining this with the fact that $A \mid Y_{1} \subset A_{0}$,

$$
A \mid Y_{1} \subset A_{0} \subset A
$$

Hence, in view of $\left(\mathrm{a}_{7}\right)$, we have $A=\bar{A}_{0}$, namely, $A$ is the complete infinitesimal generator of $\{T(t) ; t \geqq 0\}$. Finally, $X_{0}$ is dense in $X$, for $\bar{X}_{0} \supset \sum$ and $\bar{\Sigma}=X$.

Q.E.D.

We next mention a generation theorem for semi-groups of class $\left(\mathfrak{S}_{k}\right)$ which appears as a corollary of Theorem 6.2.

Theorem 6.3. Let $A$ be a densely defined, closed linear operator in a Banach space $X$ and let $k$ be a nonnegative integer. Assume that

$\left(\mathrm{d}_{1}\right)$ there is a real $\omega$ such that for each $\lambda>\omega, R(\lambda-A)$ contains $D\left(A^{k}\right)$ and $R(\lambda) \equiv(\lambda-A)^{-1}$ exists,

$\left(\mathrm{d}_{2}\right)$ there exists a constant $M>0$ such that 
$\left\|R(\lambda)^{n} x\right\| \leqq M(\lambda-\omega)^{-n}\|x\|_{k} \quad$ for $x \in D\left(A^{k}\right), \lambda>\omega$ and $n \geqq 0$

$\left(\mathrm{d}_{3}\right)$ for every $\varepsilon>0$ and $x \in D\left(A^{k}\right)$, there exist $M_{\varepsilon}>0$ and $\lambda_{0}=$ $\lambda_{0}(\varepsilon, x)$ such that

$\left\|\lambda^{n} R(\lambda)^{n} x\right\| \leqq M_{\varepsilon}\|x\| \quad$ for $\lambda>\lambda_{0}$ and $n$ with $n / \lambda \in[\varepsilon, 1 / \varepsilon]$,

( $\left.\mathrm{d}_{4}\right) \quad D\left(A^{k+1}\right)$ is $N(\cdot)$-dense in $D\left(A^{k}\right)$, where $N(\cdot)$ is defined by $N(x)$ $=\sup _{n \geqq 0, \lambda>\omega}\left\|(\lambda-\omega)^{n} R(\lambda)^{n} x\right\|$ for $x \in D\left(A^{k}\right)$,

(d $\mathrm{d}_{5} \quad D\left(A^{k+1}\right)$ is a core of $A$.

Then $A$ is the complete infinitesimal generator of a semi-group $\{T(t)$; $t \geqq 0$ \} of class $\left(\mathfrak{S}_{k}\right)$ and (6.1) holds for $x \in D\left(A^{k}\right)$ and $t \geqq 0$.

Proof. Letting $Y=D\left(A^{k}\right)$ and $\|x\|=\|x\|_{k}$ and then applying Theorem 6.2 , we see that there exists a semi-group $\{T(t) ; t \geqq 0\}$ on $X$ such that $X_{0} \equiv \cup_{t>0} T(t)[X]$ is dense in $X, A$ is its complete infinitesimal generator and such that $D\left(A^{k}\right) \subset \sum$, the continuity set of $\{T(t) ; t \geqq 0\}$. Now, it remains to show that there exists an $\omega^{\prime}>\omega_{0}$ such that for each $\lambda>\omega^{\prime}, R_{0}(\lambda)$ is closable and its closure is invertible, where $\omega_{0}$ denotes the type of $\{T(t) ; t \geqq 0\}$ and where $R_{0}(\lambda), \lambda>\omega_{0}$, are the operators on $X_{0}$ defined by (3.1). But this has already been shown in the proof of Theorem 6.2 .

Q.E.D.

Remarks. (1) Theorem 6.3 is the converse of Theorem 3.5.

(2) In case of $k=0,\left(\mathrm{~d}_{1}\right)$ states that $\{\lambda ; \lambda>\omega\}$ is contained in the resolvent set $\rho(A)$ of $A$. In this case condition $\left(\mathrm{d}_{2}\right)$ yields that $N(\cdot)$ is equivalent to $\|\cdot\|$, and hence $\left(\mathrm{d}_{3}\right)$ is automatically satisfied. Also, under conditions $\left(\mathrm{d}_{1}\right)$ and $\left(\mathrm{d}_{2}\right)$ with $k=0$, it is proved that $\left(\mathrm{d}_{4}\right)$ and $\left(\mathrm{d}_{5}\right)$ are also satisfied. Hence, Theorem 6.3 gives a generation theorem for semigroups of class $\left(C_{0}\right)$.

(3) In case of $k=1, N(x) \leqq M\|x\|_{1} \quad$ for $x \in D(A)$, and so, $\left(\mathrm{d}_{4}\right)$ follows from $\left(d_{5}\right)$.

(4) It is sometimes convenient to employ the following somewhat stronger condition $\left(\mathrm{d}_{4}{ }^{\prime}\right)$ in Theorem 6.3 , instead of $\left(\mathrm{d}_{4}\right)$ :

$\left(\mathrm{d}_{4}{ }^{\prime}\right) \quad D\left(A^{k+1}\right)$ is dense in the Banach space $\left[D\left(A^{k}\right)\right]$. 
Finally, we discuss the semi-group whose complete infinitesimal generator has nonempty resolvent set.

Corollary 6.4. Let $A$ be a densely defined, closed linear operator in X. If

$\left(\mathrm{d}_{1}{ }^{\prime}\right)$ there is a real $\omega$ such that $\{\lambda ; \lambda>\omega\} \subset \rho(A)$, and if $\left(\mathrm{d}_{2}\right)$ and $\left(\mathrm{d}_{3}\right)$ in Theorem 6.3 are satisfied, then $A$ is the complete infinitesimal generator of a semi-group of class $\left(C_{(k)}\right)$.

Proof. It is proved ([9; Lemma 2.7]) that if $A$ is a closed operator satisfying $\left(\mathrm{d}_{1}{ }^{\prime}\right)$ and if $\overline{D(A)}=X$ then $D\left(A^{n+1}\right)$ is dense in $\left[D\left(A^{n}\right)\right]$ for each nonnegative integer $n$. Hence, $\left(\mathrm{d}_{4}\right)$ and $\left(\mathrm{d}_{5}\right)$ are satisfied. Therefore, by Theorem 6.3, $A$ becomes the complete infinitesimal generator of a semi-group $\{T(t) ; t \geqq 0\}$ of class $\left(\mathfrak{S}_{k}\right)$. Now, from $\left(\mathrm{d}_{1}{ }^{\prime}\right)$ and the definition of class $\left(C_{(k)}\right)$ it follows that $\{T(t) ; t \geqq 0\}$ is of class $\left(C_{(k)}\right)$. Q.E.D.

Remark 6.5. The definition of class $\left(C_{(k)}\right)$ proposed in Oharu [9] is slightly different from the one given in Remark (1) after Definition 3.3. The relationship between those two definitions is stated as follows: A semi-group $\{T(t) ; t \geqq 0\}$ on $X$ is of class $\left(C_{(k)}\right)$ if and only if it satisfies

$\left(\alpha_{1}{ }^{\prime}\right)$ there exists an $\omega>\omega_{0}$ such that for each $\lambda>\omega$, there exists an $R(\lambda) \in B(X)$ with the properties

(a) $R(\lambda) x=\int_{0}^{\infty} e^{-\lambda t} T(t) x d t\left(=R_{0}(\lambda) x\right) \quad$ for $x \in X_{0}$,

(b) $R(\lambda)$ is invertible,

together with conditions $\left(\alpha_{2}\right)$ and $\left(\alpha_{3}\right)$ (stated in Definition 3.3).

In fact, if $\{T(t) ; t \geqq 0\}$ is of class $\left(C_{(k)}\right)$, then for each $\lambda>\omega, R(\lambda)$ is a closed operator defined on $X$ and hence it is bounded. Since (a) and (b) are trivially satisfied, condition ( $\left.\alpha_{1}^{\prime}\right)$ holds. We note that in this case $\{\lambda ; \lambda>\omega\} \subset \rho(A)$ and $R(\lambda)=R(\lambda ; A)$ for $\lambda>\omega$. Conversely, assume conditions $\left(\alpha_{1}^{\prime}\right),\left(\alpha_{2}\right)$ and $\left(\alpha_{3}\right)$. Clearly, $R_{0}(\lambda)$ is closable and $\bar{R}_{0}(\lambda) \subset R(\lambda)$ for $\lambda>\omega$. Let $x \in X$. By $\left(\alpha_{2}\right)$, a sequence $\left\{x_{n}\right\}$ can be found in $X_{0}$ such that $x_{n} \rightarrow x$ as $n \rightarrow \infty$. Making use of $\left(\alpha_{1}^{\prime}\right), R_{0}(\lambda) x_{n}=R(\lambda) x_{n} \rightarrow$ 
$R(\lambda) x$ as $n \rightarrow \infty$; this means that $\bar{R}_{0}(\lambda) x=R(\lambda) x$ for $x \in X$ and $\lambda>\omega$.

\section{Abstract Cauchy Problem}

In this section we are concerned with the abstract Cauchy problem. Let $A$ be a closed linear operator in a Banach space $X$ and let us consider a differential equation in $X$

$$
(d / d t) u(t)=A u(t), \quad t>0 .
$$

By an abstract Cauchy problem for $A$ we mean the following:

ACP. Given an element $x \in X$, find an $X$-valued function $u(t)=u(t$; $x)$, defined on $[0, \infty)$, such that

(i) $u(t)$ is continuously differentiable in $t \geqq 0$ (or $t>0$ ),

(ii) for each $t>0, u(t) \in D(A)$ and $u(t)$ satisfies (7.1)

(iii) $\lim _{t \rightarrow 0+} u(t)=u(0)=x$.

The function $u(t)$ satisfying (i)-(iii) is called the solution of ACP. There are two alternatives for condition (i); the corresponding problems will be denoted by $\mathrm{ACP}_{1}$ and $\mathrm{ACP}_{2}$, respectively. Let $D$ be a linear manifold in $X$. By a family of solution operators of $A C P$ on $D$ we mean a one-parameter family $\{U(t) ; t \geqq 0\}$ of linear operators, defined on $D$, such that for each $x \in D$ the function $u(t)=U(t) x$ is a solution of the underlying problem. Our object is to derive some sufficient conditions for such solution operators to exist uniquely. The domain $D$ of the solution operators $U(t)$ constructed in the sequel is not necessarily dense in $X$ and $U(t)$ need not be bounded on $D$ even if $\bar{D}=X$. However, as will be shown later, the results are closely related to the notions of Hadamard correctness, semi-group (S. G. -) well-posedness and well-posedness in the sense of distribution semi-group.

We divide this section into two parts and discuss the construction of solution operators of $\mathrm{ACP}_{1}$ and $\mathrm{ACP}_{2}$, separately.

Throughout this section, let $A$ be a closed linear operator in a fixed Banach space $X$ and we assume that there exists a real $\omega_{0}$ such that $R(\lambda)=(\lambda-A)^{-1}$ exists for $\lambda>\omega_{0}$. 
7.1. Let $D$ be a linear manifold in $X$ such that

(7.2) there is a norm $\|\cdot\| \|$ under which $D$ is a normed space,

(7.3) there is a seminorm $p(\cdot)$ on $D$,

and let us consider a family of operators $U(t)$ on $D$ into $D(A)$ satisfying (7.4) for every $x \in D, u(t)=U(t) x$ is a solution of $\mathrm{ACP}_{1}$ for $A$ with the initial value $u(0)=x$,

(7.5) there exists a positive constant $M$ such that

$\|U(t) x\| \leqq M e^{\omega_{0} t}\|x\|$ and $\|A U(t) x\| \leqq M e^{\omega_{0} t} p(x) \quad$ for $x \in D, t>0$.

We call this $\{U(t) ; t \geqq 0\}$ a family of solution operators of $\mathrm{ACP}_{1}$ on $D$ with type $\omega_{0}$ and $D$ the domain of the solution operators.

Remark 7.1. Since $A$ is closed, the solution $u(t)$ of $\mathrm{ACP}_{1}$ satisfy the equation (7.1) at $t=0$ and its initial value $u(0)=x$ belongs to $D(A)$. Therefore, it must hold that $D \subset D(A)$. Also, by (7.5), the norm $\|\cdot\| \|$ is stronger than the original norm $\|\cdot\|$ on $D$.

Lemma 7.2. (i) $R(\lambda-A) \supset D$ and $R(\lambda) x=\int_{0}^{\infty} e^{-\lambda t} U(t) x d t$ for $\lambda>$ $\omega_{0}$ and $x \in D$.

(ii) For each $x \in D$, the corresponding solution $u(t)=U(t) x$ is unique in the sense that $e^{-\omega_{0} t} u(t)$ is uniformly bounded on $[0, \infty)$ together with $e^{-\omega_{0} t} A u(t)$.

Proof. (i) is obtained from the relation $(d / d t)\left[e^{-\lambda t} U(t) x\right]=-e^{-\lambda t}$ $(\lambda-A) U(t) x$ for $t \geqq 0, \lambda>\omega_{0}$ and $x \in D$, and (ii) follows from (i).

Q.E.D.

In view of this lemma, the family of solution operators satisfying (7.4) and (7.5) can not exist more than one. Hence, according to KreinLaptev-Cvetkova [6] we see that $\mathrm{ACP}_{1}$ is Hadamard correct with type $\omega_{0}$ on $D$ when the family as above exists on $D$.

Now, let us consider the set $\hat{D}$ consisting of those elements $x$ for 
which there exists a solution $u(t ; x)$ of $\mathrm{ACP}_{1}$ for $A$ such that

$$
\sup _{t \geqq 0}\left\|e^{-\omega_{0} t} u(t ; x)\right\|<\infty \text { and } \sup _{t \geqq 0}\left\|e^{-\omega_{0} t} A u(t ; x)\right\|<\infty \text {. }
$$

In the same way as in Lemma 7.2, we see that the solution $u(t ; x)$ is unique in the sense that (7.6) holds. By virtue of the linearity of $A$ and Remark 7.1, $\hat{D}$ forms a linear manifold in $X$ such that $\hat{D} \subset D(A)$. Moreover, let $\omega>\omega_{0}$ be arbitrarily fixed, then $\hat{D}$ becomes a normed space under $N^{\prime}(\cdot)$ defined by

$$
N^{\prime}(x)=\sup _{t \geqq 0}\left\|e^{-\omega t} u(t ; x)\right\|(\geqq\|x\|), \quad x \in \hat{D}
$$

and $q(x)=\sup _{t \geqq 0}\left\|e^{-\omega t} A u(t ; x)\right\|, x \in \hat{D}$, define a seminorm on $\hat{D}$. Therefore, we can define linear operators $\hat{U}(t)$ from $\hat{D}$ into $D(A)$ by $\hat{U}(t) x=$ $u(t ; x), x \in \hat{D}, t \geqq 0 ;\{\hat{U}(t) ; t \geqq 0\}$ is a family of solution operators on $\hat{D}$ satisfying (7.4) and (7.5) with $D, M\|\cdot\|$ and $M p(\cdot)$ replaced by $\hat{D}$, $N^{\prime}(\cdot)$ and $q(\cdot)$, respectively. $\hat{D}$ is maximal in the sense that every domain $D$ of solution operators of $\mathrm{ACP}_{1}$ for the fixed operator $A$ satisfying (7.4) and (7.5) is contained in $\hat{D}$, note that in this case $N^{\prime}(x) \leqq M\|x\|$ and $q(x) \leqq M p(x)$ for $x \in D$. The following lemma is easily seen:

Lemma 7.3. $\hat{U}(t)$ maps $\hat{D}$ into itself and $\hat{U}(t+s)=\hat{U}(t) \hat{U}(s)$ on $\hat{D}$ for $t, s \geqq 0$. Furthermore, $N^{\prime}(\hat{U}(t) x) \leqq e^{\omega t} N^{\prime}(x)$ for $t \geqq 0$ and $x \in \hat{D}$.

Also, in the same way as in Lemma 7.2 and Feller [3; Section 3], we obtain the following (cf. $\left[6\right.$; Propositions $\left.1^{\circ}-5^{\circ}\right]$ ):

Lemma 7.4. (i) $R(\lambda-A) \supset \hat{D}$ and $R(\lambda) x=\int_{0}^{\infty} e^{-\lambda t} \hat{U}(t) x d t$ for $\lambda>$ $\omega$ and $x \in \hat{D}$.

(ii) $R(\lambda)[\hat{D}] \subset \hat{D}$ and $R(\lambda)-R(\mu)=-(\lambda-\mu) R(\lambda) R(\mu)$ on $\hat{D}$ for $\lambda, \mu>\omega$.

(iii) $\quad N^{\prime}(x)=\sup _{n \geqq 0, \lambda>\omega}\left\|(\lambda-\omega)^{n} R(\lambda)^{n} x\right\|$ and

$$
q(x)=\sup _{n \geqq 0, \lambda>\omega}\left\|(\lambda-\omega)^{n} A R(\lambda)^{n} x\right\| \quad \text { for } x \in \hat{D} .
$$

Lemma 7.4 states that the pair of $A$ and $\hat{D}$ satisfies conditions $\left(\mathrm{a}_{1}\right)$ (a a $_{3}$ with $Y=\hat{D}$ and $M\|\cdot\|=N^{\prime}(\cdot)$. We then define a linear subset $\widetilde{\Sigma}$ 
and a norm $\tilde{N}(\cdot)$ on $\widetilde{\Sigma}$ by (5.1) and (5.2); $\widetilde{\Sigma}$ is a Banach space under $\tilde{N}(\cdot)$ by Lemma 5.3. Then $\hat{D} \subset \widetilde{\Sigma}$ and $N^{\prime}(x)=\tilde{N}(x)$ for $x \in \hat{D}$ by Lemma 7.4 (iii). Since the pair of $A$ and $\widetilde{\Sigma}$ satisfies also $\left(a_{1}\right)-\left(a_{3}\right)$ with $M\|\cdot\|=\tilde{N}(\cdot)$, we see applying Remark 5.5 that there exists a semigroup $\{T(t) ; t \geqq 0\}$ of class $\left(C_{0}\right)$ on the $\tilde{N}(\cdot)$-closure $\left(\widetilde{\Sigma}_{1}\right)^{-}$of $\widetilde{\Sigma}_{1}$ such that $\tilde{N}(\tilde{T}(t) x) \leqq e^{\omega t} \tilde{N}(x)$ for $x \in\left(\widetilde{\Sigma}_{1}\right)^{-}$and $t \geqq 0$ and also that its infinitesimal generator is $\tilde{A}=\left(A \mid \widetilde{\Sigma}_{2}\right)^{-}=A \mid R(\lambda)\left[\left(\widetilde{\Sigma}_{1}\right)^{\sim}\right]$, where $\widetilde{\Sigma}_{1}=\{x \in \widetilde{\Sigma}$; $A x \in \widetilde{\Sigma}\}, \widetilde{\Sigma}_{2}=\left\{x \in \widetilde{\Sigma}_{1} ; A x \in \widetilde{\Sigma}_{1}\right\}$ and where $\left(A \mid \widetilde{\Sigma}_{2}\right)^{-}$denotes the $\tilde{N}(\cdot)$. closure of $A \mid \widetilde{\Sigma}_{2}$. Therefore, it follows that $D(\tilde{A}) \subset \hat{D}$ and $\widetilde{T}(t) \mid D(\tilde{A}) \subset$ $\hat{U}(t)$ for $t \geqq 0$. Consequently, we have

$$
\begin{aligned}
\hat{D} \supset D(\tilde{A}) & =D\left(\left(A \mid \widetilde{\Sigma}_{2}\right)^{\sim}\right)=R(\lambda)\left[\left(\widetilde{\Sigma}_{1}\right)^{\sim}\right] \\
& =\left\{x \in\left(\widetilde{\Sigma}_{1}\right)^{\sim} \cap D(A) ; A x \in\left(\widetilde{\Sigma}_{1}\right)^{\sim}\right\} .
\end{aligned}
$$

Since $N^{\prime}(x)=N(x)$ for $x \in \hat{D}$, Lemma 7.3 yields that each $\hat{U}(t)$ can be uniquely extended to an operator $\tilde{U}(t)$ on the $\tilde{N}(\cdot)$-closure $\tilde{D}$ of $\hat{D}$ such that $\tilde{N}(\tilde{U}(t) x) \leqq e^{\omega t} \tilde{N}(x)$ for $x \in \tilde{D}$. It is clear that $\tilde{U}(t)$ maps $\tilde{D}$ into itself. The relationship between $\{\tilde{T}(t) ; t \geqq 0\}$ and $\{\tilde{U}(t) ; t \geqq 0\}$ is stated as follows:

Theorem 7.5. $\tilde{U}(t)=\widetilde{T}(t)$ for $t \geqq 0$ and $\{\tilde{U}(t) ; t \geqq 0\}$ forms a semigroup of class $\left(C_{0}\right)$ on the Banach space $\check{D}$ whose infinitesimal generator $\tilde{A}$ coincides with $A \mid \hat{D}$.

Proof. Lemma 7.3 implies that $\{\tilde{U}(t) ; t \geqq 0\}$ has the semi-group property. Let $x \in \hat{D}$. Since $\left\|e^{-\omega t} \hat{U}(t) x\right\| \rightarrow 0$ as $t \rightarrow \infty$, we see from the same argument as in Theorem 2.2 that $N^{\prime}(\hat{U}(t) x-x)=\tilde{N}(\tilde{U}(t) x-x) \rightarrow 0$ as $t \rightarrow 0+$. Hence, $\{\widetilde{U}(t) ; t \geqq 0\}$ forms a semi-group of class $\left(C_{0}\right)$ on $\widetilde{D}$. Let $\tilde{A}_{1}$ be the infinitesimal generator and let $\lambda>\omega$. Then $\left(\lambda-\tilde{A}_{1}\right)^{-1} x=$ $\int_{0}^{\infty} e^{-\lambda t} \tilde{U}(t) x d t$ for $x \in \tilde{D}$. Since $R(\lambda) x=\int_{0}^{\infty} e^{-\lambda t} \hat{U}(t) x d t$ for $x \in \hat{D}$ by Lemma 7.4, $R(\lambda) x=\left(\lambda-\tilde{A}_{1}\right)^{-1} x$ for $\lambda>\omega$ and $x \in \tilde{D}$. So that $x=(\lambda-A)$ $\left(\lambda-\tilde{A}_{1}\right)^{-1} x$ for $\lambda>\omega$ provided $x \in D\left(\tilde{A}_{1}\right)$. Since $\lambda A\left(\lambda-\tilde{A}_{1}\right)^{-1} x=\lambda \tilde{A}_{1}(\lambda-$ $\left.\tilde{A}_{1}\right)^{-1} x \rightarrow \tilde{A}_{1} x$ as $\lambda \rightarrow+\infty$, the closedness of $A$ yields that $A x=\tilde{A}_{1} x$. This also means that $D\left(\tilde{A}_{1}\right) \subset \widetilde{\Sigma_{1}}$ and hence $\tilde{D} \subset\left(\widetilde{\Sigma}_{1}\right)^{\sim}$. But, $\widetilde{T}(t) \mid D(\tilde{A})$ 
$\subset \hat{U}(t) \subset \tilde{U}(t)$ for $t \geqq 0$; hence $\tilde{U}(t)=\widetilde{T}(t)$ for $t \geqq 0$. Consequently, $\tilde{A}_{1}=$ $\tilde{A}=A|R(\lambda)[\tilde{D}] \subset A| \hat{D}$. Next, we show that $\tilde{A}=A \mid \hat{D}$. Let $h_{0}>0$ and let $x \in \hat{D}$. Then $h^{-1}[\hat{U}(h) x-x]-h^{\prime-1}\left[\hat{U}\left(h^{\prime}\right) x-x\right] \in \hat{D}$ for $h, h^{\prime} \in\left(0, h_{0}\right)$. We want to show that $\tilde{N}(\cdot)-\lim _{h \rightarrow 0+} h^{-1}[\hat{U}(h) x-x]$ exists and is equal to $A x$. Noting that

$$
h^{-1}[\hat{U}(s+h) x-\hat{U}(s) x]=\int_{0}^{1} A \hat{U}(s+h \theta) x d \theta, \quad s \geqq 0, h \in\left(0, h_{0}\right),
$$

we obtain

$$
\begin{aligned}
e^{-\omega s} & \hat{U}(s)\left[h^{-1}(\hat{U}(h) x-x)-h^{-1}\left(\hat{U}\left(h^{\prime}\right) x-x\right)\right] \\
& =e^{-\omega s} \int_{0}^{1}\left[A \hat{U}(s+h \theta) x-A \hat{U}\left(s+h^{\prime} \theta\right) x\right] d \theta .
\end{aligned}
$$

Since $N_{0}(x)=\sup _{s \geqq 0}\left\|e^{-\omega_{0} s} A \hat{U}(s) x\right\|<+\infty$,

$$
\begin{gathered}
\left\|e^{-\omega s} \int_{0}^{1} A \hat{U}(s+h \theta) x d \theta\right\| \leqq N_{0}(x) e^{-\omega s} \int_{0}^{1} e^{-\omega_{0}(s+h \theta)} d \theta \\
\leqq e^{-\left(\omega-\omega_{0}\right) s} N_{0}(x) e^{\left|\omega_{0}\right| h_{0}} \rightarrow 0 \text { as } s \rightarrow+\infty .
\end{gathered}
$$

Therefore, $e^{-\omega s} \int_{0}^{1}\left[A \hat{U}(s+h \theta) x-A \hat{U}\left(s+h^{\prime} \theta\right) x\right] d \theta \rightarrow 0$ as $h, h^{\prime} \rightarrow 0$, uniformly for $s \geqq 0$, i.e., $N^{\prime}\left(h^{-1}[\hat{U}(h) x-x]-h^{\prime-1}\left[\hat{U}\left(h^{\prime}\right) x-x\right]\right) \rightarrow 0$ as $h, h^{\prime} \rightarrow$ $0+$. From this we see that

$$
\tilde{N}(\cdot)-\lim _{h \rightarrow 0+} h^{-1}[\hat{U}(h) x-x]=A x \in \tilde{D}=\left(\widetilde{\Sigma}_{1}\right)^{-} .
$$

Since $\bar{A}$ is the infinitesimal generator of the semi-group $\{\tilde{U}(t) ; t \geqq 0\}$ of class $\left(C_{0}\right)$ on $\tilde{D}$, we have that $\hat{D} \subset D(\tilde{A})$. Combining this with (7.7), we obtain $\hat{D}=D(\tilde{A})$ and hence $\tilde{A}=A \mid \hat{D}$.

Q.E.D.

Remarks. (1) The main results announced in Krein-Laptev-Cvetkova $[6 ;$ p. 766] are obtained from Lemmas 7.2, 7.4 and Theorem 7.5.

(2) We showed that $N^{\prime}(x)=\tilde{N}(x)$ for $x \in \hat{D}$. Also, the assertion of Theorem 7.5 states that $q(x)=\tilde{N}(A x)$. Therefore, we can let $q(x)=$ $\sup _{n \geq 0, \lambda>\infty}\left\|(\lambda-\omega)^{n} R(\lambda)^{n} A x\right\|$ in Lemma 7.4 (iii).

(3) Let $A$ be the complete infinitesimal generator of a semi-group 
$\{T(t) ; t \geqq 0\}$ of class $\left(\mathfrak{S}_{k}\right)$ and let us consider $\mathrm{ACP}_{1}$ for $A$. Then $D=$ $D\left(A^{k+1}\right)$ is a normed space under $\|\cdot\|=\|\cdot\|_{k}$ and $p(x)=\|A x\|_{k}, x \in D$, define a seminorm on $D$. Also, Theorem 6.2 states that $\{U(t) ; t \geqq 0\}$ defined by $U(t)=T(t) \mid D\left(A^{k+1}\right), t \geqq 0$, satisfies (7.4) and (7.5). Hence, the maximal domain $\hat{D}$ of solution operators of $\mathrm{ACP}_{1}$ for $A$ contains $D$. But, in virtue of Theorems 2.6, 6.1 and 7.5, we see that $\hat{D}$ coincides with the domain $D(\Omega)$ of the infinitesimal generator in the sense of Feller of $\{T(t) ; t \geqq 0\}$. That is, $\mathrm{ACP}_{1}$ for $A$ is Hadamard correct with type $\omega_{0}$ on $D(\Omega)$. See also Theorem 7.7 .

Next, we consider some particular cases. Let $k$ be a positive integer and assume the following conditions which are treated in Theorem 6.3:

$\left(\mathrm{d}_{1}\right) \quad R(\lambda-A) \supset D\left(A^{k}\right)$ for $\lambda>\omega$,

$\left(\mathrm{d}_{2}\right)$ there exists a constant $M>0$ such that $\left\|R(\lambda)^{n} x\right\| \leqq M(\lambda-\omega)^{-n}$ $\|x\|_{k}$ for $x \in D\left(A^{k}\right), \lambda>\omega$ and $n \geqq 0$.

We first note that $R(\lambda)^{n}\left[D\left(A^{k}\right)\right] C D\left(A^{k+n}\right)$ for $\lambda>\omega$ and $n \geqq 1$ and that the pair of $A$ and $\left[D\left(A^{k}\right)\right]$ satisfies conditions $\left(\mathrm{a}_{1}\right)-\left(\mathrm{a}_{3}\right)$. Now, let $Y=\left[D\left(A^{k}\right)\right]$ in Remark 5.5; then $Y_{1}=D\left(A^{k+1}\right)$ and $Y_{2}=D\left(A^{k+2}\right)$. Hence, there is a semi-group $\{\widetilde{T}(t) ; t \geqq 0\}$ of class $\left(C_{0}\right)$ on the $\tilde{N}(\cdot)$-closure $D\left(A^{k+1}\right)^{-}$of $D\left(A^{k+1}\right)$ such that $\widetilde{T}(t) x=\tilde{N}(\cdot)-\lim _{n \rightarrow \infty}\left(I-\frac{t}{n} A\right)^{-n} x$ and $\tilde{N}(\tilde{T}(t) x) \leqq e^{\omega t} \tilde{N}(x)$ for $t \geqq 0$ and $x \in D\left(A^{k+1}\right)$. Also, its infinitesimal generator $\tilde{A}$ coincides with $A \mid R(\lambda)\left[D\left(A^{k+1}\right)^{-}\right]$and $R(\lambda) \mid D\left(A^{k+1}\right)^{-}$is the resolvent of $\tilde{A}$ at $\lambda>\omega$. We then set

$$
U(t)=\widetilde{T}(t) \mid D\left(A^{k+1}\right) \quad \text { for } t \geqq 0 .
$$

Then it is easily seen that $\{U(t) ; t \geqq 0\} \subset B\left(\left[D\left(A^{k+1}\right)\right], X\right)$ and

(7.8) for every $x \in D\left(A^{k+1}\right), U(t) x$ is $\|\cdot\|$-continuous in $t \geqq 0$ and $\|U(t) x\|$ $\leqq \mathrm{Me}^{\omega t}\|x\|_{k}$,

$$
\begin{aligned}
& U(t) x-x=\int_{0}^{t} U(s) A x d s \quad \text { for } x \in D\left(A^{k+1+1}\right) \text { and } t \geqq 0, \\
& A^{p} U(t) x=U(t) A^{p} x \quad \text { for } \quad x \in D\left(A^{k+1+p}\right), t \geqq 0 \text { and } p \geqq 1, \\
& U(t+s) x=U(t) U(s) x \quad \text { for } \quad x \in D\left(A^{2(k+1)}\right) \text { and } s, t \geqq 0,
\end{aligned}
$$




$$
R(\lambda) x=\int_{0}^{\infty} e^{-\lambda t} U(t) x d t \quad \text { for } x \in D\left(A^{k+1}\right) \text { and } \lambda>\omega
$$

If $D\left(A^{k+1}\right)$ is $N(\cdot)$-dense in $D\left(A^{k}\right)$, where $N(\cdot)$ is defined on $D\left(A^{k}\right)$ by (4.3), then the semi-group $\{\widetilde{T}(t)\}$ is constructed on $D\left(A^{k}\right)^{-}$by Theorem 5.4, from which it follows that (7.8)-(7.12) hold for $k+1$ replaced by $k$. Consequently, we obtain

Theorem 7.6. If $\left(\mathrm{d}_{1}\right)$ and $\left(d_{2}\right)$ are satisfied for some $k \geqq 1$, then $A C P_{1}$ for $A$ is Hadamard correct with type $\omega$ on $D\left(A^{k+2}\right)$. If in addition, $D\left(A^{k+1}\right)$ is $N(\cdot)$-dense in $D\left(A^{k}\right)$, then the $A C P_{1}$ is Hadamard correct on $D\left(A^{k+1}\right)$.

Remarks. (1) If $D\left(A^{k+1}\right)$ is $N(\cdot)$-dense in $D\left(A^{k}\right)$, then so is $D\left(A^{k+n}\right)$ for $n \geq 2$.

(2) In order that $D\left(A^{k+1}\right)$ is $N(\cdot)$-dense in $D\left(A^{k}\right)$, it is sufficient that $D\left(A^{k+1}\right)$ is dense in $\left[D\left(A^{k}\right)\right]$. If $A$ is densely defined and if $\rho(A)$ $\neq \emptyset$, then $D\left(A^{k+1}\right)$ is dense in $\left[D\left(A^{k}\right)\right]$. Hence, our result extends those of Sova [13; Theorem 2.6] and Oharu [9; Theorem 4.3].

(3) A linear operator $A$ in a Banach space $X$ is the infinitesimal generator of an exponential distribution semi-group if and only if $A$ is a densely defined, closed operator in $X$ with $\rho(A) \neq \varnothing$ and satisfying $\left(\mathrm{d}_{1}\right)$ and $\left(\mathrm{d}_{2}\right)$ for some $k \geqq 0$, see [14; Theorem 3.2] and [9; Theorem 5.5].

Before concluding this subsection, we exhibit an example of an operator satisfying $\left(\mathrm{d}_{1}\right)$ and $\left(\mathrm{d}_{2}\right)$. Let $X=L^{2}(R) \times L^{2}(R)$, and let $A$ be a differential operator of the form

$$
A(D)=\left(\begin{array}{cc}
0 & 1 \\
0 & 0
\end{array}\right) D \quad D=i(\partial / \partial s)
$$

Applying the Fourier transform, the equation $(\partial / \partial t) u(t, s)=A(D) u(t, s)$ is reduced to the ordinary differential equations with $\xi$ as a parameter

$$
(d / d t) \hat{u}(t ; \xi)=A(\xi) \hat{u}(t ; \xi), \quad \xi \in R,
$$

where $\hat{u}$ denotes the Fourier transform of $u$, and 


$$
(\lambda E-A(\xi))^{-1}=\lambda^{-1} E+\lambda^{-2} A(\xi),
$$

$E$ being the $2 \times 2$ unit matrix. Moreover,

$$
\begin{aligned}
(\lambda E-A(\xi))^{-n} & =(-1)^{n-1}(n-1) !^{-1}(\partial / \partial \lambda)^{n-1}(\lambda E-A(\xi))^{-1} \\
& =\lambda^{-n} E+n \lambda^{-n-1} A(\xi) .
\end{aligned}
$$

(7.13) states that $R(\lambda-A)=D\left((\lambda-A)^{-1}\right)=D(A)$ for $\lambda>0$. By virtue of (7.14), we have that $\left\|\lambda^{n}(\lambda-A)^{-n} x\right\| \leqq\|x\|+(n / \lambda)\|A x\|$ for $\lambda>0, n \geqq 0$ and $x \in D(A)$. Therefore, $A$ satisfies $\left(\mathrm{d}_{1}\right)$ and $\left(\mathrm{d}_{2}\right)$ with $k=1$. On the other hand,

$$
e^{t A(\xi)}=E+t A(\xi), \quad \xi \in R .
$$

The solution operator $U(t)$ is obtained by taking the inverse Fourier transform of $e^{t A(\xi)}$, note that $\{U(t) ; t \geqq 0\}$ can not be extended to a semigroup on $X$. (7.15) states that $U(t)$ map $D(A)$ into itself and that $(d / d t)$ $U(t) x=A x$ for $x \in D(A)$ and $t \geqq 0$. Hence, $U(t) \subset \hat{U}(t)$ for $t \geqq 0$ and $\hat{D} \subset \widetilde{\Sigma} \subset D(A) \subset \hat{D}$, and so, $D(\tilde{A})=\hat{D}=\widetilde{D}=\widetilde{\Sigma}=D(A)$. Consequently, in this case, $\{U(t) ; t \geqq 0\}$ forms a semi-group on the Banach space $D(A)$ endowed with the norm $\tilde{N}(\cdot)$ and $A$ is its infinitesimal generator.

7.2. In this subsection we restrict ourselves to a densely defined, closed linear operator $A$ and discuss the construction of solution operators of $\mathrm{ACP}_{2}$ for $A$.

Theorem 7.7. Let $\hat{D}$ be the maximal domain of solution operators of $A C P_{1}$ for $A$ and $\{\hat{U}(t) ; t \geqq 0\}$ be the family of solution operators on $\hat{D}$. Let $\{\widetilde{U}(t) ; t \geqq 0\}$ be the semi-group obtained by Theorem 7.5 and $\tilde{A}$ be its infinitesimal generator. Assume that $\hat{D}$ be a core of $A$ and

(C) for every $t>0$ there is an $M_{t}>0$ such that

$$
\|\hat{U}(t) x\| \leqq M_{t}\|x\| \quad \text { for } x \in \hat{D} .
$$

Then $\tilde{A}(=A \mid \hat{D})$ is the infinitesimal generator in the sense of Feller and $A$ is the complete infinitesimal generator both of which are of a semi-group $\{T(t) ; t \geqq 0\}$ on $X$ such that 
(a) $T(t) \mid \tilde{D}=\tilde{U}(t)$ for $t \geqq 0$ and $\tilde{D}$ is the continuity set,

(b) $A T(t) x=T(t) A x$ and $T(t) x-T(s) x=\int_{s}^{t} T(\sigma) A x d \sigma$ for $x \in$ $D(A)$ and $t \geqq s>0$.

Therefore, $V(t) \equiv T(t) \mid \tilde{D} \cap D(A), t \geqq 0$, form a family of solution operators of $A C P_{2}$ for $A$.

Proof. Since $\hat{D}$ is dense in $X$, each $\hat{U}(t)$ admits a unique extension $T(t) \in B(X)$. In view of the fact that $\left(\widetilde{\Sigma}_{1}\right)^{-}=\tilde{D}$, we have $T(t) \mid \widetilde{D}=$ $\tilde{U}(t), t \geqq 0$. Hence, Theorem 7.5 and condition (C) imply that $\{T(t) ; t \geqq$ $0\}$ forms a semi-group on $X$. Let $x \in D(A)$. Since $\hat{D}$ is a core of $A$, there is a sequence $\left\{x_{n}\right\} \subset \hat{D}$ such that $\left\|x_{n}-x\right\|+\left\|A x_{n}-A x\right\| \rightarrow 0$; hence $A T(t) x_{n}=\tilde{A} \tilde{U}(t) x_{n}=\tilde{U}(t) \tilde{A} x_{n}=T(t) A x_{n} \rightarrow T(t) A x$ for $t>0$. From this it follows that $A T(t) x=T(t) A x$ and $T(t) x-T(s) x=\int_{s}^{t} T(\sigma) A x d \sigma$ for $x \in D(A)$ and $t \geqq s>0$. Thus, we have (b). Next, we show that $A$ is equal to the infinitesimal generator in the sense of Feller $\Omega$ of $\{T(t)$; $t \geqq 0\}$. Let $Y=\tilde{D}, A=\tilde{A}$ and let $N(\cdot)=\tilde{N}(\cdot)$ in Theorem 6.1. Theorem 7.5 states that those $Y, A$ and $N(\cdot)$ satisfy $\left(c_{1}\right)-\left(c_{3}\right)$. Also, since $\{T(t)$; $t \geqq 0\}$ is a semi-group on $X,\left(\mathrm{c}_{4}\right)$ is easily verified. Hence, Theorem 6.1 yields that $\tilde{A}=\Omega$ and that $\tilde{D}$ coincides with the continuity set of $\{T(t)$; $t \geqq 0\}$. Finally, to prove that $A$ is the complete infinitesimal generator of $\{T(t) ; t \geqq 0\}$, let $Y=\tilde{D},\|\cdot\|=\tilde{N}(\cdot)$ in Theorem 6.2. Then by assumptions, $A$ satisfies $\left(\mathrm{a}_{1}\right)$ and $\left(\mathrm{a}_{2}\right)$. Also, Theorem 7.5 states that $\left(\mathrm{a}_{3}\right)$ and $\left(\mathrm{a}_{4}\right)$ are satisfied. $\left(\mathrm{a}_{6}\right)$ follows from the fact that $T(t) \mid \tilde{D}=\tilde{U}(t), t \geqq 0$, and $\left(\mathrm{a}_{7}\right)$ is a part of the assumption. Hence, Theorem 6.2 implies that $A$ is the complete infinitesimal generator of $\{T(t) ; t \geqq 0\}$.

Q.E.D.

Remarks. We note that $D(\Omega)=\hat{D} \subset D\left(A_{0}\right) \subset \widetilde{D} \cap D(A)$ in the above theorem, see also Lemma 3.2. According to Krein [5], condition (C) is called the condition of correct posedness of ACP. As is seen from Section $6,(C)$ is equivalent to the condition of Feller type, $\left(a_{6}\right)$.

Theorem 7.8. Let $A$ be a densely defined, closed linear operator in $X$ and $k$ be a positive integer. Suppose conditions $\left(\mathrm{d}_{1}\right),\left(\mathrm{d}_{2}\right),\left(\mathrm{d}_{3}\right)$ (stated 
in Theorem 6.3) and

$\left(\mathrm{d}_{4}{ }^{\prime}\right) \quad D\left(A^{k+1}\right)$ is dense in $\left[D\left(A^{k}\right)\right]$ and $D\left(A^{k}\right)$ is dense in $X$.

Then there exist a one-parameter family $\{U(t) ; t \geqq 0\} \subset B\left(\left[D\left(A^{k}\right)\right], X\right)$ and a semi-group $\{T(t) ; t \geqq 0\}$ on $X$ such that

(a) $T(t) \mid D\left(A^{k}\right)=U(t) \quad$ for $t \geqq 0$ and $D\left(A^{k}\right)$ is contained in the continuity set,

(b) $A^{p} T(t) x=T(t) A^{p} x \quad$ for $x \in D\left(A^{k}\right), t>0$ and $p=1,2, \cdots k$,

(c) $T(t) x-T(s) x=\int_{s}^{t} T(\sigma) A x d \sigma \quad$ for $x \in D\left(A^{k}\right)$ and $t \geqq s>0$.

Therefore, $\{U(t) ; t \geqq 0\}$ becomes a family of solution operators of $A C P_{2}$ for $A$.

Proof. As was stated in Remark (2) after Theorem 7.6, $\left(\mathrm{d}_{4}{ }^{\prime}\right)$ implies that $D\left(A^{k+1}\right)$ is $N(\cdot)$-dense in $D\left(A^{k}\right)$. Hence, as is shown in the proof of Theorem 7.6, a semi-group $\{\widetilde{T}(t) ; t \geqq 0\}$ of class $\left(C_{0}\right)$ is constructed on $D\left(A^{k}\right)^{-}$. We then put $U(t)=\widetilde{T}(t) \mid D\left(A^{k}\right)$ for $t \geqq 0$. Then $\{U(t)$; $t \geqq 0\} \subset B\left(\left[D\left(A^{k}\right)\right], X\right)$. On the other hand, the pair of $A$ and $\left[D\left(A^{k}\right)\right]$ satisfies conditions $\left(\mathrm{a}_{1}\right)-\left(\mathrm{a}_{6}\right)$ with $Y=\left[D\left(A^{k}\right)\right]$ and $\|\cdot\|=\|\cdot\|_{k}$; hence by Theorem 5.6 and Theorem 7.6, a semi-group $\{T(t) ; t \geqq 0\}$ can be found such that $T(t) \mid D\left(A^{k}\right)=U(t)$ for $t \geqq 0$. In order to show (b), we observe that $D\left(A^{k+n}\right)$ is dense in $\left[D\left(A^{k}\right)\right]$ for $n \geqq 2$. In view of this, given an $x \in D\left(A^{k}\right)$, a sequence $\left\{x_{n}\right\}\left(C D\left(A^{2 k}\right)\right)$ can be found such that $x_{n} \rightarrow x$ and $A^{j} x_{n} \rightarrow A^{j} x, j=1,2, \cdots k$, from which it follows that $U(t) x_{n}$ $\rightarrow T(t) x$ and $A^{j} U(t) x_{n}=U(t) A^{j} x_{n} \rightarrow T(t) A^{j} x, j=1,2, \cdots, k$, for each $t \geqq 0$. Note that $A^{j}$ commutes with $U(t)$ on $D\left(A^{k+j}\right)$. Hence, we first have that $T(t) x \in D(A)$ and $A T(t) x=T(t) A x$ for $t>0$ and then obtain the assertion (b) inductively. Now, let $x \in D\left(A^{k}\right)$ and $\left\{x_{n}\right\} \subset D\left(A^{2 k}\right)$ be a sequence as above. Then we see from the same argument as in (7.9) that $U(t) x_{n}-U(s) x_{n}=\int_{s}^{t} U(\sigma) A x_{n} d \sigma$ for $t \geqq s>0$. Passing to the limit as $n \rightarrow \infty$, we obtain

$$
T(t) x-T(s) x=\int_{s}^{t} T(\sigma) A x d \sigma, \quad x \in D\left(A^{k}\right)
$$


Since $T(t) A x$ is $\|\cdot\|$-continuous in $s>0,(7.16)$ means that $T(t) x$ is a solution of $\mathrm{ACP}_{2}$ associated with the initial value $x$.

Q.E.D.

Remarks. (1) If $x \in D\left(A^{k}\right)$, then $u(t)=T(t) x$ gives a unique solution of $\mathrm{ACP}_{2}$ for $A$ in the sense that $u(t) \in D\left(A^{k}\right)$ for $t>0$. In fact, let $0<s<t$. Then $(d / d s)[T(t-s+\varepsilon) u(s)]=-T(t-s+\varepsilon) A u(s)+T(t-s+\varepsilon)$ $A u(s)=0$. Integrating this from $s=\delta>0$ to $s=t-\delta$, we obtain $T(\delta+\varepsilon)$ $u(t-\delta)=T(t-\delta+\varepsilon) u(\delta)$. Now, letting $\delta \rightarrow 0+$, we obtain $T(\varepsilon) u(t)=$ $T(t+\varepsilon) x$; hence it follows that $u(t)=T(t) x$ for $t \geqq 0$.

(2) Let $A$ be a densely defined, closed linear operator and suppose that there exists a family of operators $U(t)$, defined on a core $D$ of $A$, such that for any $x \in D, u(t)=U(t) x$ is a unique solution of $\mathrm{ACP}_{1}$ for $A$ with the initial value $x$ and $u(t) \in D$ for $t>0$. When each $U(t)$ is bounded on $D, U(t)$ admits an extension $T(t) \in B(X)$ and $\{T(t) ; t \geqq 0\}$ becomes a semi-group on $X$. In this way, if there is a semi-group $\{T(t)$; $t \geqq 0\}$ on $X$ such that $T(t) \mid D=U(t)$ for $t \geqq 0$, then we say that ACP is well-posed in the sense of semi-group on $X$ (simply, S.G.-well-posed). If the semi-group is of class $\left(\Im_{k}\right)$ for some nonnegative integer $k$, then we say that the ACP is $\left(\mathfrak{S}_{k}\right)$-well-posed. Theorem 7.7 gives a sufficient condition for ACP to be S.G.-well-posed and Theorem 7.8 gives a sufficient condition for ACP to be $\left(\mathfrak{S}_{k}\right)$-well-posed. The $\left(C_{(k)}\right)$-well-posedness and the well-posedness in the sense of distribution semi-group are similarly defined (cf. Remark (3) after Theorem 7.6); for details, see TakahashiOharu [16; Section 4].

Finally, we make mention of an example of the operator satisfying $\left(\mathrm{d}_{1}\right)-\left(\mathrm{d}_{4}{ }^{\prime}\right)$. Let $X=L^{2}(R) \times L^{2}(R)$ and $A$ be a differential operator of the form

$$
A(D)=\left(\begin{array}{cc}
-1 & 0 \\
0 & -1
\end{array}\right) D^{2}+\left(\begin{array}{ll}
0 & 1 \\
0 & 0
\end{array}\right) D^{2 r}, \quad D=i(\partial / \partial s)
$$

where $r$ is a nonnegative integer. Applying the Fourier transform, $A(D)$ is converted to a multiplication operator in $X$ 


$$
A(\xi)=\left(\begin{array}{cc}
-\xi^{2} & \xi^{2 r} \\
0 & -\xi^{2}
\end{array}\right), \quad \xi \in R \text {. }
$$

Then, for each $\lambda>0, \lambda E-A(\xi)$ has the inverse

$$
(\lambda E-A(\xi))^{-1}=\left(\lambda+\xi^{2}\right)^{-1}\left(\lambda+2 \xi^{2}\right) E+\left(\lambda+\xi^{2}\right)^{-2} A(\xi) .
$$

Hence, if $r \geqq 3,(\lambda-A)^{-1}$ is not bounded for $\lambda>0$ and $\rho(A)=\emptyset$. But we see from (7.17) that $R(\lambda-A)=D\left((\lambda-A)^{-1}\right) \supset D(A)$ for $\lambda>0$. On the other hand, for a fixed $\xi \in R$, we have

$$
(\lambda E-A(\xi))^{-n}=\left(\lambda+\xi^{2}\right)^{-n} E+n \xi^{2 r}\left(\lambda+\xi^{2}\right)^{-(n+1)} F,
$$

where $F=\left(\begin{array}{ll}0 & 1 \\ 0 & 0\end{array}\right)$. Hence, we have

$$
\left\|\lambda^{n}(\lambda-A)^{-n} x\right\| \leqq\left[1+(n / \lambda)^{1-r}(n r /(n+1-r))^{r}(1-r /(n+1))^{n+1}\right]\|x\|
$$

for $\lambda>0, n \geqq r$ and $x \in X$; this estimate shows that $\left(\mathrm{d}_{3}\right)$ holds. Moreover, (7.18) can be written as

$$
(\lambda E-A(\xi))^{-n}=\left[(n+1) \xi^{2}+\lambda\right]\left(\lambda+\xi^{2}\right)^{-n-1} E+n\left(\lambda+\xi^{2}\right)^{-n-1} A(\xi)
$$

and hence we have that $\left\|\lambda^{n}(\lambda-A)^{-n} x\right\| \leqq\|x\|+(n / \lambda)\|A x\|$ for $\lambda>0, n \geqq 0$ and $x \in D(A)$. To see that condition $\left(\mathrm{d}_{4}{ }^{\prime}\right)$ holds, it suffices to observe that $C_{0}^{\infty}(R) \times C_{0}^{\infty}(R)$ is contained in $D\left(A^{2}\right)$ and dense in $[D(A)]$. Thus, we obtain a semi-group $\{T(t) ; t \geqq 0\}$ on $X$ of class $\left(\Im_{1}\right)$ such that $A$ is the complete infinitesimal generator and such that $\|T(t)\| \leqq 1+(r / e)^{r} t^{1-r}$ for $t>0$ and $\|T(t) x\| \leqq\|x\|+t\|A x\|$ for $x \in D(A)$ and $t \geqq 0$.

\section{References}

[1] Crandall, M. and T. Liggett, Generation of semi-groups of nonlinear transformations on general Banach spaces, Amer. J. Math. 93 (2) (1971), 265-298.

[2] Da Prato, G., Nouveau-type de semi-groupes, C.R. Acad. Sci. Paris Ser. A-B, 262 (1966), A996-A998.

[3] Feller, W., On the generation of unbounded semi-groups of bounded linear operators, Ann. of Math. 58 (2) (1953), 166-174.

[4] Hille, E. and R. Phillips, Functional Analysis and Semi-Groups, Amer. Math. Soc, Colloq. Publ. 31 (1957). 
[5] Krein, S., Linear Differential Equations in Banach Spaces, Nauka, 1967 (in Russian).

[6] Krein, S., G. Laptev and G. Cvetkova, On Hadamard correctness of the Cauchy problem for the equation of evolution, Soviet Math. Dokl. 11 (3) (1970), 763-766.

[7] Lagnese, J., On equations of evolution and parabolic equation of higher order in t, J. Math. Anal. Appl. 32 (1970), 15-37.

[8] Miyadera, I., On the generation of semi-groups of linear operators, Tôhoku Math. J. 24 (1972), 251-261.

[9] Oharu, S., Semigroups of linear operators in a Banach space, Publ. RIMS, Kyoto Univ. 7 (2) (1971), 205-260.

[10] Okazawa, N., A generation theorem for semi-groups of growth order $\alpha$, Tôhoku Math. J. 25 (1973), to appear.

[11] Phillips, R., An inversion formula for Laplace transforms and semi-groups of linear operators, Ann. of Math. 59 (2) (1954), 325-356.

[12] Sobolevskii, P. On semigroups of growth $\alpha$, Soviet Math. Dokl. 12 (1), (1971), 202-205.

[13] Sova, M., Problème de Cauchy pour équations hyperboliques opérationnelles à coefficients non-bornes, Ann. Scuola Norm. Sup. Pisa, 22 (1968), 67-100.

[14] — Problèmes de Cauchy paraboliques abstraits de classes supérieures et les semi-groupes distributions, Ricerche Mat. 18 (1969), 215-238.

[15] Sunouchi, H., Convergence of semi-discrete difference schemes of Cauchy problems, Tôhoku Math. J. 22 (1970), 394-408.

[16] Takahashi, T. and S. Oharu, Approximation of operator semigroups in a Banach space, Tôhoku Math. J. 24 (1972), 505-528.

[17] Yosida, K., Functional Analysis, Springer, Berlin, 1965. 
